\title{
INTUITIONISTIC FUZZY EINSTEIN CHOQUET INTEGRAL OPERATORS FOR MULTIPLE ATTRIBUTE DECISION MAKING
}

\author{
Yejun XU ${ }^{\mathrm{a}, \mathrm{b}}$, Huimin WANG ${ }^{\mathrm{a}, \mathrm{b}}$, José M. MERIGÓc, d \\ aState Key Laboratory of Hydrology-Water Resources and Hydraulic Engineering, Hohai University, \\ 210098 Nanjing, PR China \\ ${ }^{\mathrm{b}}$ Research Institute of Management Science, Business School, Hohai University, \\ 211100 Nanjing, PR China \\ ${ }^{c}$ Department of Business Administration, University of Barcelona, \\ Av. Diagonal 690, 08034 Barcelona, Spain \\ ${ }^{\mathrm{d}}$ Manchester Business School, The University of Manchester, \\ Booth Street West, M15 6PB Manchester, United Kingdom
}

Received 28 July 2012; accepted 01 June 2013

\begin{abstract}
In this paper, we propose some new aggregation operators which are based on the Choquet integral and Einstein operations. The operators not only consider the importance of the elements or their ordered positions, but also consider the interactions phenomena among the decision making criteria or their ordered positions. It is shown that the proposed operators generalize several intuitionistic fuzzy Einstein aggregation operators. Moreover, some of their properties are investigated. We also study the relationship between the proposed operators and the existing intuitionistic fuzzy Choquet aggregation operators. Furthermore, an approach based on intuitionistic fuzzy Einstein Choquet integral operators is presented for multiple attribute decision-making problem. Finally, a practical decision making problem involving the water resource management is given to illustrate the multiple attribute decision making process.
\end{abstract}

Keywords: Einstein operations, intuitionistic fuzzy set, fuzzy measure, intuitionistic fuzzy Einstein Choquet aggregation operators.

Reference to this paper should be made as follows: Xu, Y.; Wang, H.; Merigó, J. M. 2014. Intuitionistic fuzzy Einstein Choquet integral operators for multiple attribute decision making, Technological and Economic Development of Economy 20(2): 227-253.

JEL Classification: A12, C44, C60, D81.

Corresponding author Huimin Wang

E-mail: hmwang@hhu.edu.cn 


\section{Introduction}

The concept of intuitionistic fuzzy set (IFS) was introduced by Atanassov $(1986,1999)$ to generalize the concept of Zadeh's fuzzy set (Zadeh 1965). Each element in IFS is expressed by an ordered pair, and each ordered pair is characterized by a membership degree and a non-membership degree. The sum of the membership degree and non-membership degree of each ordered pair is less than or equal to one. In the following several decades, IFS theory has been widely studied and developed. In the early of its appearance, many papers paid attention on the basic concept of the IFS such as operations on IFSs (De et al. 2000), distances between IFSs (Chen 2007; Grzegorzewski 2004; Szmidt, Kacprzyk 2000), similarity measures between IFSs (Chen 1997; Hung, Yang 2004; Li, Cheng 2002; Liang, Shi 2003), correlation of IFSs (Bustince, Burillo 1995; Hong, Hwang 1995), etc. Recently, some approaches were investigated to multiple attribute decision making (MADM) problems based on IFSs (Li 2005; Lin et al. 2007; Xu et al. 2010; Xu 2011). Many aggregation operators of IFSs are proposed, such as intuitionistic fuzzy weighted geometric (IFWG) operator (Xu, Yager 2006), intuitionistic fuzzy ordered weighted geometric (IFOWG) operator (Xu, Yager 2006), intuitionistic fuzzy hybrid geometric (IFHG) operator (Xu, Yager 2006), intuitionistic fuzzy weighted averaging (IFWA) operator (Xu 2007), intuitionistic fuzzy ordered weighted averaging (IFOWA) operator (Xu 2007), intuitionistic fuzzy hybrid averaging (IFHA) operator (Xu 2007), dynamic intuitionistic fuzzy weighted averaging (DIFWA) operator (Wei 2009), dynamic intuitionistic fuzzy weighted geometric (DIFWG) operator, induced intuitionistic fuzzy ordered weighted geometric (I-IFOWG) operator (Wei 2010), generalized intuitionistic fuzzy weighted averaging (GIFWA) operator (Zhao et al. 2010), generalized intuitionistic fuzzy ordered weighted averaging (GIFOWA) operator, and generalized intuitionistic fuzzy hybrid averaging (GIFHA) operator, induced generalized intuitionistic fuzzy ordered weighted averaging (I-GIFOWA) operator (Xu, Wang 2012), etc. All the above mentioned intuitionistic fuzzy aggregation operators only consider situations where all the elements in an IFS are independent, i.e. they only consider the addition of the importance of individual elements. However, in many practical situations, the elements in an IFS are usually correlative. The Choquet integral (Choquet 1954) is a very useful way of measuring the expected utility of an uncertain event, and can be used to depict the correlations of the data under consideration. Based on the correlation properties of the Choquet integral, Xu (2010), Tan and Chen (2010) almost simultaneously proposed the intuitionistic fuzzy Choquet integral operator, respectively. All the above operators are based on the algebraic operational laws of IFSs for carrying the combination process and are not consistent with the limiting case of ordinary fuzzy sets (Beliakov et al. 2011). Recently, Wang and Liu $(2011,2012)$ developed some intuitionistic fuzzy aggregation operators based on Einstein operations. Einstein operations include Einstein product and Einstein sum, which are good alternatives to the algebraic product and algebraic sum, respectively. Therefore, extending the Einstein operations to aggregate the intuitionistic fuzzy information is a meaningful work, which is also the focus of this paper.

The remainder of this paper is organized as follows. In Section 1, we briefly reviews some basic concepts related to the IFSs, fuzzy measure and some existing intuitionistic fuzzy Choquet operators. In Section 2, we introduce the Einstein operations and extend them to 
the intuitionistic fuzzy operations. Based on these intuitionistic fuzzy Einstein operations and fuzzy measure, we develop some new aggregation operators, such as intuitionistic fuzzy Einstein Choquet averaging $\left(\mathrm{IFCA}^{\varepsilon}\right)$ operator, intuitionistic fuzzy Einstein Choquet geometric $\left(\mathrm{IFCG}^{\varepsilon}\right.$ ) operator, and study various special cases of the operators, and also investigate some desired properties of the developed operators, such as commutativity, idempotency, boundary, etc. Furthermore, we compare these operators with the existing intuitionistic fuzzy averaging operators. We also develop a procedure for multi-attribute decision making. In Section 4, we apply the developed operators to decision making problem with intuitionistic fuzzy information. The final section ends this paper with some concluding remarks.

\section{Preliminaries}

In 1986, Atanassov (1986) generalized the concept of Zadeh's fuzzy set (Zadeh 1965), and defined the concept of intuitionistic fuzzy set as follows.

Given a fixed set $X=\left\{x_{1}, x_{2}, \ldots, x_{n}\right\}$, an IFS is defined as:

$$
A=\left\{<x, \mu_{A}(x), v_{A}(x)>\mid x \in X\right\},
$$

which is characterized by a membership function $\mu_{A}: X \rightarrow[0,1]$ and a non-membership function $v_{A}: X \rightarrow[0,1]$, with the condition:

$$
0 \leq \mu_{A}(x)+v_{A}(x) \leq 1, \forall x \in X,
$$

where the numbers $\mu_{A}(x)$ and $v_{A}(x)$ represent, respectively, the degree of membership and the degree of non-membership of the element $x$ to the set $A$.

For each IFS $A$ in $X$, if:

$$
\pi_{A}(x)=1-\mu_{A}(x)-v_{A}(x), \forall x \in X,
$$

is called the indeterminacy degree or hesitation degree of $x$ to $A$. Especially, if:

$$
\pi_{A}(x)=1-\mu_{A}(x)-v_{A}(x)=0, \forall x \in X,
$$

then, the IFS $A$ is reduced to a common fuzzy set.

For convenience, $\mathrm{Xu}$ and Yager (2006) called $\alpha=\left(\mu_{\alpha}, v_{\alpha}\right)$ an intuitionistic fuzzy value (IFV), where $\mu_{\alpha} \in[0,1], v_{\alpha} \in[0,1]$, and $\mu_{\alpha}+v_{\alpha} \leq 1$. For convenience, let $\Omega$ be the set of all IFVs.

Let $\alpha=\left(\mu_{\alpha}, v_{\alpha}\right)$ be an IFV, Chen and Tan (1994) introduced a score function $S$, which can be represented as follows:

$$
S(\alpha)=\mu_{\alpha}-v_{\alpha}
$$

where $S(\alpha) \in[-1,1]$.

For an IFV $\alpha=\left(\mu_{\alpha}, v_{\alpha}\right)$, it is clear that if the deviation between $\mu_{\alpha}$ and $v_{\alpha}$ gets greater, which means the value $\mu_{\alpha}$ gets bigger and the value $v_{\alpha}$ gets smaller, then the IFV $\alpha$ gets greater.

Later, Hong and Choi (2000) noted that the score function alone cannot differentiate many IFVs even though they are obviously different. To make the comparison method more 
discriminatory, an accuracy function $H$ to evaluate the degree of accuracy of the IFV can be represented as follows:

$$
H(\alpha)=\mu_{\alpha}+v_{\alpha}
$$

where $H(\alpha) \in[0,1]$. The larger the value of $H(\alpha)$, the higher the degree of accuracy of the degree of membership of the IFV $\alpha$.

As presented above, the score function $S$ and the accuracy function $H$ are, respectively, defined as the difference and the sum of the membership function $\mu_{A}(x)$ and the non-membership function $v_{A}(x)$. Xu and Yager (2006) showed that the relationship between the score function $S$ and the accuracy function $H$ is similar to the relation between mean and variance in statistics. Based on the score function $S$ and the accuracy function $H$, Xu and Yager (2006) introduced an order relation between two intuitionistic fuzzy numbers in the following:

Definition 1 (Xu, Yager 2006). Let $\alpha=\left(\mu_{\alpha}, v_{\alpha}\right)$ and $\beta=\left(\mu_{\beta}, v_{\beta}\right)$ betwo IFVs, $S(\alpha)=\mu_{\alpha}-v_{\alpha}$ and $S(\beta)=\mu_{\beta}-v_{\beta}$ be the scores of $\alpha$ and $\beta$, respectively, and let $H(\alpha)=\mu_{\alpha}+v_{\alpha}$ and $H(\beta)=$ $\mu_{\beta}+v_{\beta}$ be the accuracy degrees of $\alpha$ and $\beta$, then:

1. If $S(\alpha)<S(\beta)$, then $\alpha$ is smaller than $\beta$, denoted by $\alpha<\beta$.

2. If $S(\alpha)=S(\beta)$, then:

(1) If $H(\alpha)=H(\beta)$, then $\alpha$ and $\beta$ represent the same information, i.e. $\mu_{\alpha}=\mu_{\beta}, v_{\alpha}=v_{\beta}$, denoted by $\alpha=\beta$;

(2) If $H(\alpha)<H(\beta)$, then $\alpha$ is smaller than $\beta$, denoted by $\alpha<\beta$.

To aggregate intuitionistic preference information, $\mathrm{Xu}$ (2007) defined the following operations:

Definition 2 (Xu 2007). Let $\alpha=\left(\mu_{\alpha}, v_{\alpha}\right)$ and $\beta=\left(\mu_{\beta}, v_{\beta}\right)$ be two IFVs, then:

(1) $\alpha \oplus \beta=\left(\mu_{\alpha}+\mu_{\beta}-\mu_{\alpha} \cdot \mu_{\beta}, v_{\alpha} \cdot v_{\beta}\right)$;

(2) $\alpha \otimes \beta=\left(\mu_{\alpha} \cdot \mu_{\beta}, v_{\alpha}+v_{\beta}-v_{\alpha} \cdot v_{\beta}\right)$;

(3) $\lambda \alpha=\left(1-\left(1-\mu_{\alpha}\right)^{\lambda}, v_{\alpha}^{\lambda}\right), \lambda>0$;

(4) $\alpha^{\lambda}=\left(\mu_{\alpha}^{\lambda}, 1-\left(1-v_{\alpha}\right)^{\lambda}\right), \lambda>0$.

In 1974, Sugeno (1974) introduced the concept of fuzzy measure (non-additive measure), which only make a monotonicity instead of additive property. For decision making problems, it does not need an assumption that criteria or preferences are independent of one another, and was used as a powerful tool for modeling interaction phenomena in decision making. As an aggregation operator, the Choquet integral has been proposed by many authors as an adequate substitute to the weighted arithmetic mean or OWA (Yager 1988) operator to aggregate interacting criteria. In the Choquet integral model, where criteria can be dependent, a fuzzy measure is used to define a weight on each combination of criteria, thus making it possible to model the interaction existing among criteria.

Definition 3 (Wang, Klir 1992). A fuzzy measure $m$ on the set $X$ is a set function $m$ : $P(X) \rightarrow[0,1]$ satisfying the following axioms:

(1) $m(\varnothing)=0, m(X)=1$;

(2) $A \subseteq B$ implies $m(A) \leq m(B)$, for all $A, B \subseteq X$. 
Sugeno (1974) proposed a special kind of fuzzy measure defined on $P(X)$ and satisfying the finite $\rho$-rule, which satisfies the following additional property:

(3) $m(A \cup B)=m(A)+m(B)+\rho m(A) m(B)$, for all $A, B \in P(X)$, and $A \cap B=\varnothing, \rho>-1$.

In particular, if $\rho=0$, then the condition (3) reduces to the axiom of additive measure:

$$
m(A \cup B)=m(A)+m(B) \text {, for all } A, B \subseteq X \text { and } A \cap B=\varnothing .
$$

In this case, all the elements in $X$ are independent, and we have:

$$
m(A)=\sum_{x_{i} \in A} m\left(\left\{x_{i}\right\}\right)
$$

If $\rho>0$, then $m(A \cup B)>m(A)+m(B)$, which implies that the set $\{A, B\}$ has multiplicative effect. If $\rho<0$, then $m(A \cup B)<m(A)+m(B)$, which implies that the set $\{A, B\}$ has substitutive effect. By parameter $\rho$, the interaction between sets or elements of set can be represented.

Let $X=\left\{x_{1}, x_{2}, \ldots, x_{n}\right\}$ be a finite set, then $\bigcup_{i=1}^{n} x_{i}=X$. To determine normalized measure on $X$ avoiding the computational complexity, Sugeno (1974) gave the following equation:

$$
m(X)=m\left(\bigcup_{i=1}^{n} x_{i}\right)=\left\{\begin{array}{ll}
\frac{1}{\rho}\left(\prod_{i=1}^{n}\left[1+\rho m\left(x_{i}\right)\right]-1\right), & \text { if } \rho \neq 0 \\
\sum_{i=1}^{n} m\left(x_{i}\right), & \text { if } \rho=0
\end{array} .\right.
$$

Especially, for every subset $A \subseteq X$, we have:

$$
m(A)=\left\{\begin{array}{ll}
\frac{1}{\rho}\left(\prod_{x_{i} \in A}\left[1+\rho m\left(x_{i}\right)\right]-1\right), & \text { if } \rho \neq 0 \\
\sum_{x_{i} \in A} m\left(x_{i}\right), & \text { if } \rho=0
\end{array} .\right.
$$

Based on Eq. (9), the value $\rho$ can be uniquely determined from $m(X)=1$, which is equivalent to solving:

$$
\rho+1=\prod_{i=1}^{n}\left(1+\rho m\left(x_{i}\right)\right) .
$$

Based on Definition 3, Xu (2010) developed the IFCA operator and IFCG operator for aggregating IFVs with correlative weights as follows:

Definition 4 (Xu 2010). Let $\alpha_{j}=\left(\mu_{\alpha_{j}}, v_{\alpha_{j}}\right)(j=1,2, \ldots, n)$ be a collection of intuitionistic fuzzy values on $X$, and $m$ be a fuzzy measure on $X$, then we call

$$
\begin{aligned}
& \operatorname{IFCA}_{m}\left(\alpha_{1}, \alpha_{2}, \ldots, \alpha_{n}\right)=\left(m\left(A_{\sigma(1)}\right)-m\left(A_{\sigma(0)}\right)\right) \alpha_{\sigma(1)} \oplus\left(m\left(A_{\sigma(2)}\right)-m\left(A_{\sigma(1)}\right)\right) \alpha_{\sigma(2)} \\
& \oplus \cdots \oplus\left(m\left(A_{\sigma(n)}\right)-m\left(A_{\sigma(n-1)}\right)\right) \alpha_{\sigma(n)}= \\
& \left(1-\prod_{j=1}^{n}\left(1-\mu_{\alpha_{\sigma(j)}}\right)^{m\left(A_{\sigma(j)}\right)-m\left(A_{\sigma(j-1)}\right)}, \prod_{j=1}^{n}\left(v_{\alpha_{\sigma(j)}}\right)^{m\left(A_{\sigma(j)}\right)-m\left(A_{\sigma(j-1)}\right)}\right)
\end{aligned}
$$

an intuitionistic fuzzy correlated averaging (IFCA) operator, where $(\sigma(1), \sigma(2), \ldots, \sigma(n))$ is a permutation of $(1,2, \ldots, n)$ such that $\alpha_{\sigma(j-1)} \geq \alpha_{\sigma(j)}$ for all $j=2,3, \ldots, n, A_{\sigma(j)}=\left\{x_{\sigma(1)}, x_{\sigma(2)}, \ldots, x_{\sigma(j)}\right\}$ and $A_{\sigma(0)}=\varnothing$. 
Definition $5(\mathrm{Xu} 2010)$. Let $\alpha_{j}=\left(\mu_{\alpha_{j}}, v_{\alpha_{j}}\right)(j=1,2, \ldots, n)$ be a collection of IFVs on $X$, and $m$ be a fuzzy measure on $X$, then we call

$$
\begin{aligned}
& \operatorname{IFCG}_{m}\left(\alpha_{1}, \alpha_{2}, \ldots, \alpha_{n}\right)=\left(\alpha_{\sigma(1)}\right)^{\left(m\left(A_{\sigma(1)}\right)-m\left(A_{\sigma(0)}\right)\right)} \otimes\left(\alpha_{\sigma(2)}\right)^{\left(m\left(A_{\sigma(2)}\right)-m\left(A_{\sigma(1)}\right)\right)} \\
& \otimes \ldots \otimes\left(\alpha_{\sigma(n)}\right)^{\left(m\left(A_{\sigma(n)}\right)-m\left(A_{\sigma(n-1)}\right)\right)}= \\
& \left(\prod_{j=1}^{n}\left(\mu_{\alpha_{\sigma(j)}}\right)^{m\left(A_{\sigma(j)}\right)-m\left(A_{\sigma(j-1)}\right)}, 1-\prod_{j=1}^{n}\left(1-v_{\alpha_{\sigma(j)}}\right)^{m\left(A_{\sigma(j)}\right)-m\left(A_{\sigma(j-1)}\right)}\right)
\end{aligned}
$$

an intuitionistic fuzzy correlated geometric (IFCG) operator, where $(\sigma(1), \sigma(2), \ldots, \sigma(n))$ is a permutation of $(1,2, \ldots, n)$, such that $\alpha_{\sigma(j-1)} \geq \alpha_{\sigma(j)}, A_{\sigma(j)}=\left\{x_{\sigma(1)}, x_{\sigma(2)}, \ldots, x_{\sigma(n)}\right\}$, for $j \geq 1, A_{\sigma(0)}=\varnothing$.

\section{Intuitionistic fuzzy Einstein Choquet operators}

\subsection{Einstein operation}

Einstein product $\dot{\varepsilon}$ is a t-norm and Einstein sum $\varepsilon$ is a t-conorm, where:

$$
a \dot{\varepsilon} b=\frac{a \cdot b}{1+(1-a) \cdot(1-b)}, a \varepsilon b=\frac{a+b}{1+a \cdot b}, \forall(a, b) \in[0,1]^{2} .
$$

Based on the Einstein operations, Wang and Liu $(2011,2012)$ introduced the Einstein product and Einstein sum of IFS, respectively, as follows:

$$
\begin{gathered}
A \otimes_{\varepsilon} B=\left\{\left\langle x, \frac{\mu_{A}(x) \cdot \mu_{B}(x)}{1+\left(1-\mu_{A}(x)\right) \cdot\left(1-\mu_{B}(x)\right)}, \frac{v_{A}(x)+v_{B}(x)}{1+v_{A}(x) \cdot v_{B}(x)}\right\rangle \mid x \in X\right\} ; \\
A \oplus_{\varepsilon} B=\left\{\left\langle x, \frac{\mu_{A}(x)+\mu_{B}(x)}{1+\mu_{A}(x) \cdot \mu_{B}(x)}, \frac{v_{A}(x) \cdot v_{B}(x)}{1+\left(1-v_{A}(x)\right) \cdot\left(1-v_{B}(x)\right)}\right\rangle \mid x \in X\right\} ; \\
n \cdot{ }_{\varepsilon} A=\left\{\left\langle x, \frac{\left(1+\mu_{A}(x)\right)^{n}-\left(1-\mu_{A}(x)\right)^{n}}{\left(1+\mu_{A}(x)\right)^{n}+\left(1-\mu_{A}(x)\right)^{n}}, \frac{2\left(v_{A}(x)\right)^{n}}{\left(2-v_{A}(x)\right)^{n}+\left(v_{A}(x)\right)^{n}}\right\rangle \mid x \in X\right\} .
\end{gathered}
$$

Definition 6 (Wang, Liu 2011). If $\alpha=\left(\mu_{\alpha}, v_{\alpha}\right), \beta=\left(\mu_{\beta}, v_{\beta}\right)$ are two IFVs, then we define some new operations of IFVs based on Einstein operations as follows:

(1) $\alpha \oplus_{\varepsilon} \beta=\left(\frac{\mu_{\alpha}+\mu_{\beta}}{1+\mu_{\alpha} \mu_{\beta}}, \frac{v_{\alpha} v_{\beta}}{1+\left(1-v_{\alpha}\right)\left(1-v_{\beta}\right)}\right)$;

(2) $\alpha \otimes_{\varepsilon} \beta=\left(\frac{\mu_{\alpha} \mu_{\beta}}{1+\left(1-\mu_{\alpha}\right)\left(1-\mu_{\beta}\right)}, \frac{v_{\alpha}+v_{\beta}}{1+v_{\alpha} v_{\beta}}\right)$;

(3) $\alpha^{\wedge} \varepsilon^{\lambda}=\left(\frac{2 \mu_{\alpha}^{\lambda}}{\left(2-\mu_{\alpha}\right)^{\lambda}+\mu_{\alpha}^{\lambda}}, \frac{\left(1+v_{\alpha}\right)^{\lambda}-\left(1-v_{\alpha}\right)^{\lambda}}{\left(1+v_{\alpha}\right)^{\lambda}+\left(1-v_{\alpha}\right)^{\lambda}}\right)$.

Furthermore, we have:

(4) $\lambda \cdot{ }_{\varepsilon} \alpha=\left(\frac{\left(1+\mu_{\alpha}\right)^{\lambda}-\left(1-\mu_{\alpha}\right)^{\lambda}}{\left(1+\mu_{\alpha}\right)^{\lambda}+\left(1-\mu_{\alpha}\right)^{\lambda}}, \frac{2\left(v_{\alpha}\right)^{\lambda}}{\left(2-v_{\alpha}\right)^{\lambda}+\left(v_{\alpha}\right)^{\lambda}}\right), \lambda>0$. 
By the Einstein operational laws of intuitionistic fuzzy values, we have:

Theorem 1. Let $\alpha=\left(\mu_{\alpha}, v_{\alpha}\right), \beta=\left(\mu_{\beta}, v_{\beta}\right)$ and $\gamma=\left(\mu_{\gamma}, v_{\gamma}\right)$ be three IFVs, then:

(1) $\alpha \oplus_{\varepsilon} \beta=\beta \oplus_{\varepsilon} \alpha$;

(2) $\left(\alpha \oplus_{\varepsilon} \beta\right) \oplus_{\varepsilon} \gamma=\alpha \oplus_{\varepsilon}\left(\beta \oplus_{\varepsilon} \gamma\right)$;

(3) $\left(\alpha \otimes_{\varepsilon} \beta\right) \otimes_{\varepsilon} \gamma=\alpha \otimes_{\varepsilon}\left(\beta \otimes_{\varepsilon} \gamma\right)$;

(4) $\lambda \cdot{ }_{\varepsilon}\left(\alpha \oplus_{\varepsilon} \beta\right)=\lambda \cdot{ }_{\varepsilon} \alpha \oplus_{\varepsilon} \lambda \cdot{ }_{\varepsilon} \beta, \lambda>0$;

(5) $\left(\lambda_{1}\right){ }_{\varepsilon} \alpha \oplus_{\varepsilon}\left(\lambda_{2}\right){ }_{\varepsilon} \alpha=\left(\lambda_{1}+\lambda_{2}\right){ }_{\varepsilon} \alpha, \lambda_{1}, \lambda_{2}>0$.

Theorem 2. Let $\alpha=\left(\mu_{\alpha}, v_{\alpha}\right), \beta=\left(\mu_{\beta}, v_{\beta}\right), \gamma=\left(\mu_{\gamma}, v_{\gamma}\right), \varphi=\left(\mu_{\varphi}, v_{\varphi}\right)$ be four IFVs, then:

(1) If $\lambda_{1}>\lambda_{2}$, then $\left(\lambda_{1}\right){ }_{\varepsilon} \alpha \geq\left(\lambda_{2}\right){ }_{\varepsilon} \alpha, \alpha^{\wedge_{\varepsilon}\left(1-\lambda_{1}\right)} \geq \alpha^{\wedge_{\varepsilon}\left(1-\lambda_{2}\right)}, 0<\lambda_{1}, \lambda_{2} \leq 1$;

(2) If $\mu_{\alpha} \geq \mu_{\beta}, v_{\alpha} \leq v_{\beta}$, then $\lambda \cdot{ }_{\varepsilon} \alpha \geq \lambda \cdot{ }_{\varepsilon} \beta, \alpha^{\wedge} \varepsilon_{\varepsilon} \geq \beta^{\wedge} \lambda, 0<\lambda \leq 1$;

(3) If $\mu_{\alpha} \geq \mu_{\gamma}, \mu_{\beta} \geq \mu_{\varphi}, v_{\alpha} \leq v_{\gamma}, v_{\beta} \leq v_{\varphi}$, then $\alpha \oplus_{\varepsilon} \beta \geq \gamma \oplus_{\varepsilon} \varphi, \alpha \otimes_{\varepsilon} \beta \geq \gamma \otimes_{\varepsilon} \varphi$.

Proof. (1) Let $f(x)=\frac{a^{x}-b^{x}}{a^{x}+b^{x}}, x \in[0,1], a \geq b$, then $f^{\prime}(x)=\frac{2 a^{x} b^{x}(\ln a-\ln b)}{\left(a^{x}+b^{x}\right)^{2}} \geq 0$, i.e. $f(x)$ is an increasing function.

Thus, if $\lambda_{1}>\lambda_{2}$, then

$$
\begin{aligned}
& \frac{\left(1+\mu_{\alpha}\right)^{\lambda_{1}}-\left(1-\mu_{\alpha}\right)^{\lambda_{1}}}{\left(1+\mu_{\alpha}\right)^{\lambda_{1}}+\left(1-\mu_{\alpha}\right)^{\lambda_{1}}} \geq \frac{\left(1+\mu_{\alpha}\right)^{\lambda_{2}}-\left(1-\mu_{\alpha}\right)^{\lambda_{2}}}{\left(1+\mu_{\alpha}\right)^{\lambda_{2}}+\left(1-\mu_{\alpha}\right)^{\lambda_{2}}} ; \\
& \frac{\left(1+v_{\alpha}\right)^{1-\lambda_{1}}-\left(1-v_{\alpha}\right)^{1-\lambda_{1}}}{\left(1+v_{\alpha}\right)^{1-\lambda_{1}}+\left(1-v_{\alpha}\right)^{1-\lambda_{1}}} \leq \frac{\left(1+v_{\alpha}\right)^{1-\lambda_{2}}-\left(1-v_{\alpha}\right)^{1-\lambda_{2}}}{\left(1+v_{\alpha}\right)^{1-\lambda_{2}}+\left(1-v_{\alpha}\right)^{1-\lambda_{2}}} .
\end{aligned}
$$

Let $g(x)=\frac{a^{x}}{(2-a)^{x}+a^{x}}, x \in[0,1], a \in[0,1]$, then $g^{\prime}(x)=\frac{a^{x}(2-a)^{x}(\ln a-\ln (2-a))}{\left[(2-a)^{x}+a^{x}\right]^{2}} \leq 0$, i.e. $g(x)$ is a decreasing function.

Thus, if $\lambda_{1}>\lambda_{2}$, then:

$$
\begin{aligned}
& \frac{2\left(v_{\alpha}\right)^{\lambda_{1}}}{\left(2-v_{\alpha}\right)^{\lambda_{1}}+\left(v_{\alpha}\right)^{\lambda_{1}}} \leq \frac{2\left(v_{\alpha}\right)^{\lambda_{2}}}{\left(2-v_{\alpha}\right)^{\lambda_{2}}+\left(v_{\alpha}\right)^{\lambda_{2}}} ; \\
& \frac{2\left(\mu_{\alpha}\right)^{1-\lambda_{1}}}{\left(2-\mu_{\alpha}\right)^{1-\lambda_{1}}+\left(\mu_{\alpha}\right)^{1-\lambda_{1}}} \geq \frac{2\left(\mu_{\alpha}\right)^{1-\lambda_{2}}}{\left(2-\mu_{\alpha}\right)^{1-\lambda_{2}+\left(\mu_{\alpha}\right)^{1-\lambda_{2}}}},
\end{aligned}
$$

therefore,

$$
\begin{aligned}
& \frac{\left(1+\mu_{\alpha}\right)^{\lambda_{1}}-\left(1-\mu_{\alpha}\right)^{\lambda_{1}}}{\left(1+\mu_{\alpha}\right)^{\lambda_{1}}+\left(1-\mu_{\alpha}\right)^{\lambda_{1}}}-\frac{2\left(v_{\alpha}\right)^{\lambda_{1}}}{\left(2-v_{\alpha}\right)^{\lambda_{1}}+\left(v_{\alpha}\right)^{\lambda_{1}}} \geq \frac{\left(1+\mu_{\alpha}\right)^{\lambda_{2}}-\left(1-\mu_{\alpha}\right)^{\lambda_{2}}}{\left(1+\mu_{\alpha}\right)^{\lambda_{2}}+\left(1-\mu_{\alpha}\right)^{\lambda_{2}}}-\frac{2\left(v_{\alpha}\right)^{\lambda_{2}}}{\left(2-v_{\alpha}\right)^{\lambda_{2}}+\left(v_{\alpha}\right)^{\lambda_{2}}} \\
& \frac{2\left(\mu_{\alpha}\right)^{1-\lambda_{1}}}{\left(2-\mu_{\alpha}\right)^{1-\lambda_{1}}+\left(\mu_{\alpha}\right)^{1-\lambda_{1}}}-\frac{\left(1+v_{\alpha}\right)^{1-\lambda_{1}}-\left(1-v_{\alpha}\right)^{1-\lambda_{1}}}{\left(1+v_{\alpha}\right)^{1-\lambda_{1}}+\left(1-v_{\alpha}\right)^{1-\lambda_{1}}} \geq \frac{2\left(\mu_{\alpha}\right)^{1-\lambda_{2}}}{\left(2-\mu_{\alpha}\right)^{1-\lambda_{2}}+\left(\mu_{\alpha}\right)^{1-\lambda_{2}}}-\frac{\left(1+v_{\alpha}\right)^{1-\lambda_{2}}-\left(1-v_{\alpha}\right)^{1-\lambda_{2}}}{\left(1+v_{\alpha}\right)^{1-\lambda_{2}}+\left(1-v_{\alpha}\right)^{1-\lambda_{2}}} .
\end{aligned}
$$

By Definition 1, we have $\left(\lambda_{1}\right){ }_{\varepsilon} \alpha \geq\left(\lambda_{2}\right){ }_{\varepsilon} \alpha, \alpha^{\wedge_{\varepsilon}\left(1-\lambda_{1}\right)} \geq \alpha^{\wedge_{\varepsilon}\left(1-\lambda_{2}\right)}$.

(2) Let $f(x)=\frac{(1+x)^{a}-(1-x)^{a}}{(1+x)^{a}+(1-x)^{a}}, x \in[0,1], a \in[0,1]$, then $f^{\prime}(x)=\frac{4 a(1+x)^{a-1}(1-x)^{a-1}}{\left[(1+x)^{a}+(1-x)^{a}\right]^{2}} \geq 0$, i.e. $f(x)$ is an increasing function. 
Thus, if $\mu_{\alpha} \geq \mu_{\beta}, v_{\alpha} \leq v_{\beta}$, then:

$$
\begin{aligned}
& \frac{\left(1+\mu_{\alpha}\right)^{\lambda}-\left(1-\mu_{\alpha}\right)^{\lambda}}{\left(1+\mu_{\alpha}\right)^{\lambda}+\left(1-\mu_{\alpha}\right)^{\lambda}} \geq \frac{\left(1+\mu_{\beta}\right)^{\lambda}-\left(1-\mu_{\beta}\right)^{\lambda}}{\left(1+\mu_{\beta}\right)^{\lambda}+\left(1-\mu_{\beta}\right)^{\lambda}} ; \\
& \frac{\left(1+v_{\alpha}\right)^{\lambda}-\left(1-v_{\alpha}\right)^{\lambda}}{\left(1+v_{\alpha}\right)^{\lambda}+\left(1-v_{\alpha}\right)^{\lambda}} \leq \frac{\left(1+v_{\beta}\right)^{\lambda}-\left(1-v_{\beta}\right)^{\lambda}}{\left(1+v_{\beta}\right)^{\lambda}+\left(1-v_{\beta}\right)^{\lambda}} .
\end{aligned}
$$

Let $g(x)=\frac{x^{a}}{(2-x)^{a}+x^{a}}, x \in[0,1], a \in[0,1]$, then $g^{\prime}(x)=\frac{2 a x^{a-1}(2-x)^{a-1}}{\left[(2-x)^{a}+x^{a}\right]} \geq 0$, i.e. $g(x)$ is an increasing function.

Thus, if $\mu_{\alpha} \geq \mu_{\beta}, v_{\alpha} \leq v_{\beta}$, then:

$$
\begin{aligned}
& \frac{2\left(v_{\alpha}\right)^{\lambda}}{\left(2-v_{\alpha}\right)^{\lambda}+\left(v_{\alpha}\right)^{\lambda}} \leq \frac{2\left(v_{\beta}\right)^{\lambda}}{\left(2-v_{\beta}\right)^{\lambda}+\left(v_{\beta}\right)^{\lambda}} ; \\
& \frac{2 \mu_{\alpha}^{\lambda}}{\left(2-\mu_{\alpha}\right)^{\lambda}+\mu_{\alpha}^{\lambda}} \geq \frac{2 \mu_{\beta}^{\lambda}}{\left(2-\mu_{\beta}\right)^{\lambda}+\mu_{\beta}^{\lambda}} .
\end{aligned}
$$

Therefore,

$$
\begin{aligned}
& \frac{\left(1+\mu_{\alpha}\right)^{\lambda}-\left(1-\mu_{\alpha}\right)^{\lambda}}{\left(1+\mu_{\alpha}\right)^{\lambda}+\left(1-\mu_{\alpha}\right)^{\lambda}}-\frac{2\left(v_{\alpha}\right)^{\lambda}}{\left(2-v_{\alpha}\right)^{\lambda}+\left(v_{\alpha}\right)^{\lambda}} \geq \frac{\left(1+\mu_{\beta}\right)^{\lambda}-\left(1-\mu_{\beta}\right)^{\lambda}}{\left(1+\mu_{\beta}\right)^{\lambda}+\left(1-\mu_{\beta}\right)^{\lambda}}-\frac{2\left(v_{\beta}\right)^{\lambda}}{\left(2-v_{\beta}\right)^{\lambda}+\left(v_{\beta}\right)^{\lambda}} ; \\
& \frac{2 \mu_{\alpha}^{\lambda}}{\left(2-\mu_{\alpha}\right)^{\lambda}+\mu_{\alpha}^{\lambda}}-\frac{\left(1+v_{\alpha}\right)^{\lambda}-\left(1-v_{\alpha}\right)^{\lambda}}{\left(1+v_{\alpha}\right)^{\lambda}+\left(1-v_{\alpha}\right)^{\lambda}} \geq \frac{2 \mu_{\beta}^{\lambda}}{\left(2-\mu_{\beta}\right)^{\lambda}+\mu_{\beta}^{\lambda}}-\frac{\left(1+v_{\beta}\right)^{\lambda}-\left(1-v_{\beta}\right)^{\lambda}}{\left(1+v_{\beta}\right)^{\lambda}+\left(1-v_{\beta}\right)^{\lambda}} .
\end{aligned}
$$

By Definition 1, we have:

$\lambda \cdot{ }_{\varepsilon} \alpha \geq \lambda \cdot{ }_{\varepsilon} \beta, \alpha^{\wedge} \lambda \geq \beta^{\wedge} \lambda$.

(3) If $\mu_{\alpha} \geq \mu_{\gamma}, \mu_{\beta} \geq \mu_{\varphi}, v_{\alpha} \leq v_{\gamma}, v_{\beta} \leq v_{\varphi}$, then:

$$
\left(\mu_{\alpha}-\mu_{\gamma}\right)\left(1-\mu_{\beta} \mu_{\varphi}\right)+\left(\mu_{\beta}-\mu_{\varphi}\right)\left(1-\mu_{\alpha} \mu_{\gamma}\right) \geq 0,
$$

i.e.

$$
\frac{\mu_{\alpha}+\mu_{\beta}}{1+\mu_{\alpha} \mu_{\beta}} \geq \frac{\mu_{\gamma}+\mu_{\varphi}}{1+\mu_{\gamma} \mu_{\varphi}}
$$

and

$$
\begin{aligned}
& v_{\alpha} v_{\beta}\left(1-v_{\gamma}\right) \leq v_{\gamma} v_{\varphi}\left(1-v_{\alpha}\right), v_{\alpha} v_{\beta}\left(1-v_{\varphi}\right) \leq v_{\gamma} v_{\varphi}\left(1-v_{\beta}\right) \Leftrightarrow \\
& v_{\alpha} v_{\beta}\left(1-v_{\gamma}\right)+v_{\alpha} v_{\beta}\left(1-v_{\varphi}\right) \leq v_{\gamma} v_{\varphi}\left(1-v_{\alpha}\right)+v_{\gamma} v_{\varphi}\left(1-v_{\beta}\right) \Leftrightarrow \\
& v_{\alpha} v_{\beta}\left(1-v_{\gamma}\right)+v_{\alpha} v_{\beta}\left(1-v_{\varphi}\right)+v_{\alpha} v_{\beta} v_{\gamma} v_{\varphi} \leq v_{\gamma} v_{\varphi}\left(1-v_{\alpha}\right)+v_{\gamma} v_{\varphi}\left(1-v_{\beta}\right)+v_{\alpha} v_{\beta} v_{\gamma} v_{\varphi} \Leftrightarrow \\
& v_{\alpha} v_{\beta}\left(1+\left(1-v_{\gamma}\right)\left(1-v_{\varphi}\right)\right) \leq v_{\gamma} v_{\varphi}\left(1+\left(1-v_{\alpha}\right)\left(1-v_{\beta}\right)\right)
\end{aligned}
$$


i.e.

$$
\frac{v_{\alpha} v_{\beta}}{1+\left(1-v_{\alpha}\right)\left(1-v_{\beta}\right)} \leq \frac{v_{\gamma} v_{\varphi}}{1+\left(1-v_{\gamma}\right)\left(1-v_{\varphi}\right)}
$$

Thus,

$$
\frac{\mu_{\alpha}+\mu_{\beta}}{1+\mu_{\alpha} \mu_{\beta}}-\frac{v_{\alpha} v_{\beta}}{1+\left(1-v_{\alpha}\right)\left(1-v_{\beta}\right)} \geq \frac{\mu_{\gamma}+\mu_{\varphi}}{1+\mu_{\gamma} \mu_{\varphi}}-\frac{v_{\gamma} v_{\varphi}}{1+\left(1-v_{\gamma}\right)\left(1-v_{\varphi}\right)} .
$$

By Definition 1, we have:

$\alpha \oplus_{\varepsilon} \beta \geq \gamma \oplus_{\varepsilon} \varphi$.

Similarly, we have:

$$
\begin{aligned}
& \frac{\mu_{\alpha} \mu_{\beta}}{1+\left(1-\mu_{\alpha}\right)\left(1-\mu_{\beta}\right)} \geq \frac{\mu_{\gamma} \mu_{\varphi}}{1+\left(1-\mu_{\gamma}\right)\left(1-\mu_{\varphi}\right)} \\
& \frac{v_{\alpha}+v_{\beta}}{1+v_{\alpha} v_{\beta}} \leq \frac{v_{\gamma}+v_{\varphi}}{1+v_{\gamma} v_{\varphi}} .
\end{aligned}
$$

Thus,

$$
\frac{\mu_{\alpha} \mu_{\beta}}{1+\left(1-\mu_{\alpha}\right)\left(1-\mu_{\beta}\right)}-\frac{v_{\alpha}+v_{\beta}}{1+v_{\alpha} v_{\beta}} \geq \frac{\mu_{\gamma} \mu_{\varphi}}{1+\left(1-\mu_{\gamma}\right)\left(1-\mu_{\varphi}\right)}-\frac{v_{\gamma}+v_{\varphi}}{1+v_{\gamma} v_{\varphi}} .
$$

By Definition 1, we have:

$\alpha \otimes_{\varepsilon} \beta \geq \gamma \otimes_{\varepsilon} \varphi$.

\subsection{Intuitionistic fuzzy Einstein Choquet averaging operator}

Based on the Einstein operational laws of intuitionistic fuzzy values and Choquet integral, in what follows we develop some new operators for aggregating IFVs with correlative weights:

Definition 7. Let $\alpha_{j}=\left(\mu_{\alpha_{j}}, v_{\alpha_{j}}\right)(j=1,2, \ldots, n)$ be a collection of IFVs on $X$, and $m$ be a fuzzy measure on $X$, then we call

$$
\begin{aligned}
\operatorname{IFCA}_{m}^{\varepsilon}\left(\alpha_{1}, \alpha_{2}, \ldots, \alpha_{n}\right)= & \left(m\left(A_{\sigma(1)}\right)-m\left(A_{\sigma(0)}\right)\right){ }_{\varepsilon} \alpha_{\sigma(1)} \oplus_{\varepsilon}\left(m\left(A_{\sigma(2)}\right)-m\left(A_{\sigma(1)}\right)\right){ }_{\varepsilon} \alpha_{\sigma(2)} \\
& \oplus_{\varepsilon} \cdots \oplus_{\varepsilon}\left(m\left(A_{\sigma(n)}\right)-m\left(A_{\sigma(n-1)}\right)\right){ }_{\varepsilon} \alpha_{\sigma(n)}
\end{aligned}
$$

an intuitionistic fuzzy Einstein Choquet averaging (IFCA ${ }^{\varepsilon}$ ) operator, where $(\sigma(1), \sigma(2), \ldots, \sigma(n))$ is a permutation of $(1,2, \ldots, n)$ such that $\alpha_{\sigma(j-1)} \geq \alpha_{\sigma(j)}$ for all $j=2,3, \ldots, n$, $A_{\sigma(j)}=\left\{x_{\sigma(1)}, x_{\sigma(2)}, \ldots, x_{\sigma(j)}\right\}$ and $A_{\sigma(0)}=\varnothing$. 
Theorem 3. Let $\alpha_{j}=\left(\mu_{\alpha_{j}}, v_{\alpha_{j}}\right)(j=1,2, \ldots, n)$ be a collection of IFVs on $X$, and $m$ be a fuzzy measure on $X$, then their aggregated value by using IFCA $^{\varepsilon}$ operator is also an IFV, and $\operatorname{IFCA}_{m}^{\varepsilon}\left(\alpha_{1}, \alpha_{2}, \ldots, \alpha_{n}\right)=$

$$
\begin{aligned}
& \left(\frac{\prod_{j=1}^{n}\left(1+\mu_{\alpha_{\sigma(j)}}\right)^{m\left(A_{\sigma(j)}\right)-m\left(A_{\sigma(j-1)}\right)}-\prod_{j=1}^{n}\left(1-\mu_{\alpha_{\sigma(j)}}\right)^{m\left(A_{\sigma(j)}\right)-m\left(A_{\sigma(j-1)}\right)}}{\prod_{j=1}^{n}\left(1+\mu_{\alpha_{\sigma(j)}}\right)^{m\left(A_{\sigma(j)}\right)-m\left(A_{\sigma(j-1)}\right)}+\prod_{j=1}^{n}\left(1-\mu_{\alpha_{\sigma(j)}}\right)^{m\left(A_{\sigma(j)}\right)-m\left(A_{\sigma(j-1)}\right)}},\right. \\
& \left.\frac{2 \prod_{j=1}^{n} v_{\alpha_{\sigma(j)}}^{m\left(A_{\sigma(j)}\right)-m\left(A_{\sigma(j-1)}\right)}}{\prod_{j=1}^{n}\left(2-v_{\alpha_{\sigma(j)}}\right)^{m\left(A_{\sigma(j)}\right)-m\left(A_{\sigma(j-1)}\right)}+\prod_{j=1}^{n} v_{\alpha_{\sigma(j)}}^{m\left(A_{\sigma(j)}\right)-m\left(A_{\sigma(j-1)}\right)}}\right),
\end{aligned}
$$

where $(\sigma(1), \sigma(2), \ldots, \sigma(n))$ is a permutation of $(1,2, \ldots, n)$ such that $\alpha_{\sigma(j-1)} \geq \alpha_{\sigma(j)}$ for all $j=2,3, \ldots, n, A_{\sigma(j)}=\left\{x_{\sigma(1)}, x_{\sigma(2)}, \ldots, x_{\sigma(j)}\right\}$ and $A_{\sigma(0)}=\varnothing$.

Proof. Let $\omega_{j}=m\left(A_{\sigma(j)}\right)-m\left(A_{\sigma(j-1)}\right)$.

First, we prove that Eq. (6) is also an IFV.

Obviously,

$$
0 \leq \frac{\prod_{j=1}^{n}\left(1+\mu_{\alpha_{\sigma(j)}}\right)^{\omega_{j}}-\prod_{j=1}^{n}\left(1-\mu_{\alpha_{\sigma(j)}}\right)^{\omega_{j}}}{\prod_{j=1}^{n}\left(1+\mu_{\alpha_{\sigma(j)}}\right)^{\omega_{j}}+\prod_{j=1}^{n}\left(1-\mu_{\alpha_{\sigma(j)}}\right)^{\omega_{j}}}+\frac{2 \prod_{j=1}^{n} v_{\alpha_{\sigma(j)}}^{\omega_{j}}}{\prod_{j=1}^{n}\left(2-v_{\alpha_{\sigma(j)}}\right)^{\omega_{j}}+\prod_{j=1}^{n} v_{\alpha_{\sigma(j)}}^{\omega_{j}}}
$$

and

$$
\begin{aligned}
& \mu_{\alpha_{\sigma(j)}}+v_{\alpha_{\sigma(j)}} \leq 1 \Leftrightarrow \\
& v_{\alpha_{\sigma(j)}}+\mu_{\alpha_{\sigma(j)}} v_{\alpha_{\sigma(j)}} \leq 2-v_{\alpha_{\sigma(j)}}-2 \mu_{\alpha_{\sigma(j)}}+\mu_{\alpha_{\sigma(j)}} v_{\alpha_{\sigma(j)}} \Leftrightarrow \\
& \left(1+\mu_{\alpha_{\sigma(j)}}\right) v_{\alpha_{\sigma(j)}} \leq\left(1-\mu_{\alpha_{\sigma(j)}}\right)\left(2-v_{\alpha_{\sigma(j)}}\right) \Leftrightarrow \\
& 2\left(\prod_{j=1}^{n}\left(1+\mu_{\alpha_{\sigma(j)}}\right)^{\omega_{j}}\right)\left(\prod_{j=1}^{n} v_{\alpha_{\sigma(j)}}^{\omega_{j}}\right) \leq 2\left(\prod_{j=1}^{n}\left(1-\mu_{\alpha_{\sigma(j)}}\right)^{\omega_{j}}\right)\left(\prod_{j=1}^{n}\left(2-v_{\alpha_{\sigma(j)}}\right)^{\omega_{j}}\right) \Leftrightarrow \\
& \left(\prod_{j=1}^{n}\left(1+\mu_{\alpha_{\sigma(j)}}\right)^{\omega_{j}}\right)\left(\prod_{j=1}^{n}\left(2-v_{\alpha_{\sigma(j)}}\right)^{\omega_{j}}\right)+\left(\prod_{j=1}^{n}\left(1+\mu_{\alpha_{\sigma(j)}}\right)^{\omega_{j}}\right)\left(\prod_{j=1}^{n} v_{\alpha_{\sigma(j)}}^{\omega_{j}}\right)- \\
& \left(\prod_{j=1}^{n}\left(1-\mu_{\alpha_{\sigma(j)}}\right)^{\omega_{j}}\right)\left(\prod_{j=1}^{n}\left(2-v_{\alpha_{\sigma(j)}}\right)^{\omega_{j}}\right)-\left(\prod_{j=1}^{n}\left(1-\mu_{\alpha_{\sigma(j)}}\right)^{\omega_{j}}\right)\left(\prod_{j=1}^{n} v_{\alpha_{\sigma(j)}}^{\omega_{j}}\right)+ \\
& 2\left(\prod_{j=1}^{n}\left(1+\mu_{\alpha_{\sigma(j)}}\right)^{\omega_{j}}\right)\left(\prod_{j=1}^{n} v_{\alpha_{\sigma(j)}}^{\omega_{j}}\right)+2\left(\prod_{j=1}^{n}\left(1-\mu_{\alpha_{\sigma(j)}}\right)^{\omega_{j}}\right)\left(\prod_{j=1}^{n} v_{\alpha_{\sigma(j)}}^{\omega_{j}}\right) \leq \\
& \left(\prod_{j=1}^{n}\left(1+\mu_{\alpha_{\sigma(j)}}\right)^{\omega_{j}}\right)\left(\prod_{j=1}^{n}\left(2-v_{\alpha_{\sigma(j)}}\right)^{\omega_{j}}\right)+\left(\prod_{j=1}^{n}\left(1+\mu_{\alpha_{\sigma(j)}}\right)^{\omega_{j}}\right)\left(\prod_{j=1}^{n} v_{\alpha_{\sigma(j)}}^{\omega_{j}}\right)+ \\
& \left(\prod_{j=1}^{n}\left(1-\mu_{\alpha_{\sigma(j)}}\right)^{\omega_{j}}\right)\left(\prod_{j=1}^{n}\left(2-v_{\alpha_{\sigma(j)}}\right)^{\omega_{j}}\right)+\left(\prod_{j=1}^{n}\left(1-\mu_{\alpha_{\sigma(j)}}\right)^{\omega_{j}}\right)\left(\prod_{j=1}^{n} v_{\alpha_{\sigma(j)}}^{\omega_{j}}\right) \Leftrightarrow \\
& \left(\prod_{j=1}^{n}\left(1+\mu_{\alpha_{\sigma(j)}}\right)^{\omega_{j}}-\prod_{j=1}^{n}\left(1-\mu_{\alpha_{\sigma(j)}}\right)^{\omega_{j}}\right)\left(\prod_{j=1}^{n}\left(2-v_{\alpha_{\sigma(j)}}\right)^{\omega_{j}}+\prod_{j=1}^{n} v_{\alpha_{\sigma(j)}}^{\omega_{j}}\right)+
\end{aligned}
$$




$$
\begin{aligned}
& \left(\prod_{j=1}^{n}\left(1+\mu_{\alpha_{\sigma(j)}}\right)^{\omega_{j}}+\prod_{j=1}^{n}\left(1-\mu_{\alpha_{\sigma(j)}}\right)^{\omega_{j}}\right)\left(2 \prod_{j=1}^{n} v_{\alpha_{\sigma(j)}}^{\omega_{j}}\right) \leq \\
& \left(\prod_{j=1}^{n}\left(1+\mu_{\alpha_{\sigma(j)}}\right)^{\omega_{j}}+\prod_{j=1}^{n}\left(1-\mu_{\alpha_{\sigma(j)}}\right)^{\omega_{j}}\right)\left(\prod_{j=1}^{n}\left(2-v_{\alpha_{\sigma(j)}}\right)^{\omega_{j}}+\prod_{j=1}^{n} v_{\alpha_{\sigma(j)}}^{\omega_{j}}\right) \Leftrightarrow \\
& \frac{\prod_{j=1}^{n}\left(1+\mu_{\alpha_{\sigma(j)}}\right)^{\omega_{j}}-\prod_{j=1}^{n}\left(1-\mu_{\alpha_{\sigma(j)}}\right)^{\omega_{j}}}{\prod_{j=1}^{n}\left(1+\mu_{\alpha_{\sigma(j)}}\right)^{\omega_{j}}+\prod_{j=1}^{n}\left(1-\mu_{\alpha_{\sigma(j)}}\right)^{\omega_{j}}}+\frac{2 \prod_{j=1}^{n} v_{\alpha_{\sigma(j)}}^{\omega_{j}}}{\prod_{j=1}^{n}\left(2-v_{\alpha_{\sigma(j)}}\right)^{\omega_{j}}+\prod_{j=1}^{n} v_{\alpha_{\sigma(j)}}^{\omega_{j}}} \leq 1 \Leftrightarrow \\
& \frac{\prod_{j=1}^{n}\left(1+\mu_{\alpha_{\sigma(j)}}\right)^{\omega_{j}}-\prod_{j=1}^{n}\left(1-\mu_{\alpha_{\sigma(j)}}\right)^{\omega_{j}}}{\prod_{j=1}^{n}\left(1+\mu_{\alpha_{\sigma(j)}}\right)^{\omega_{j}}+\prod_{j=1}^{n}\left(1-\mu_{\alpha_{\sigma(j)}}\right)^{\omega_{j}}}+\frac{2 \prod_{j=1}^{n} v_{\alpha_{\sigma(j)}}^{\omega_{j}}}{\prod_{j=1}^{n}\left(2-v_{\alpha_{\sigma(j)}}\right)^{\omega_{j}}+\prod_{j=1}^{n} v_{\alpha_{\sigma(j)}}^{\omega_{j}} \leq 1 .}
\end{aligned}
$$

Thus, the aggregated value by using the $\mathrm{IFCA}^{\varepsilon}$ operator is also an IFV.

Below we prove Eq. (19) by using mathematical induction on $n$.

For $n=2$, according to operational laws of Definition 6, we have:

$$
\begin{aligned}
& \left(\omega_{1}\right)_{\varepsilon} \alpha_{\sigma(1)}=\left(\frac{\left(1+\mu_{\alpha_{\sigma(1)}}\right)^{\omega_{1}}-\left(1-\mu_{\alpha_{\sigma(1)}}\right)^{\omega_{1}}}{\left(1+\mu_{\alpha_{\sigma(1)}}\right)^{\omega_{1}}+\left(1-\mu_{\alpha_{\sigma(1)}}\right)^{\omega_{1}}}, \frac{2\left(v_{\alpha_{\sigma(1)}}\right)^{\omega_{1}}}{\left(2-v_{\alpha_{\sigma(1)}}\right)^{\omega_{1}}+\left(v_{\alpha_{\sigma(1)}}\right)^{\omega_{1}}}\right) ; \\
& \left(\omega_{2}\right)_{\varepsilon} \alpha_{\sigma(2)}=\left(\frac{\left(1+\mu_{\alpha_{\sigma(2)}}\right)^{\omega_{2}}-\left(1-\mu_{\alpha_{\sigma(2)}}\right)^{\omega_{2}}}{\left(1+\mu_{\alpha_{\sigma(2)}}\right)^{\omega_{2}}+\left(1-\mu_{\alpha_{\sigma(2)}}\right)^{\omega_{2}}}, \frac{2\left(v_{\alpha_{\sigma(2)}}\right)^{\omega_{2}}}{\left(2-v_{\alpha_{\sigma(2)}}\right)^{\omega_{2}}+\left(v_{\alpha_{\sigma(2)}}\right)^{\omega_{2}}}\right) .
\end{aligned}
$$

Then:

$$
\begin{aligned}
& \operatorname{IFCA}_{\omega}^{\varepsilon}\left(\alpha_{1}, \alpha_{2}\right)=\left(\omega_{1}\right){ }_{\varepsilon} \alpha_{\sigma(1)} \oplus_{\varepsilon}\left(\omega_{2}\right) \cdot_{\varepsilon} \alpha_{\sigma(2)}= \\
& \left(\frac{\left(1+\mu_{\alpha_{\sigma(1)}}\right)^{\omega_{1}}\left(1+\mu_{\alpha_{\sigma(2)}}\right)^{\omega_{2}}-\left(1-\mu_{\alpha_{\sigma(1)}}\right)^{\omega_{1}}\left(1-\mu_{\alpha_{\sigma(2)}}\right)^{\omega_{2}}}{\left(1+\mu_{\alpha_{\sigma(1)}}\right)^{\omega_{1}}\left(1+\mu_{\alpha_{\sigma(2)}}\right)^{\omega_{2}}+\left(1-\mu_{\alpha_{\sigma(1)}}\right)^{\omega_{1}}\left(1-\mu_{\alpha_{\sigma(2)}}\right)^{\omega_{2}}}, \frac{2 v_{\alpha_{\sigma(1)}}^{\omega_{1}} v_{\alpha_{\sigma(2)}}^{\omega_{2}}}{\left(2-v_{\alpha_{\sigma(1)}}\right)^{\omega_{1}}\left(2-v_{\alpha_{\sigma(2)}}\right)^{\omega_{2}}+v_{\alpha_{\sigma(1)}}^{\omega_{1}} v_{\alpha_{\sigma(2)}}^{\omega_{2}}}\right) .
\end{aligned}
$$

That is, for $n=2$, Eq. (19) holds.

Suppose that if for $n=k$, the Eq. (19) holds, i.e.:

$\operatorname{IFCA}_{\omega}^{\varepsilon}\left(\alpha_{1}, \alpha_{2}, \ldots, \alpha_{k}\right)=\left(\frac{\prod_{j=1}^{k}\left(1+\mu_{\alpha_{\sigma(j)}}\right)^{\omega_{j}}-\prod_{j=1}^{k}\left(1-\mu_{\alpha_{\sigma(j)}}\right)^{\omega_{j}}}{\prod_{j=1}^{k}\left(1+\mu_{\alpha_{\sigma(j)}}\right)^{\omega_{j}}+\prod_{j=1}^{k}\left(1-\mu_{\alpha_{\sigma(j)}}\right)^{\omega_{j}}}, \frac{2 \prod_{j=1}^{k} v_{\alpha_{\sigma(j)}}^{\omega_{j}}}{\prod_{j=1}^{k}\left(2-v_{\alpha_{\sigma(j)}}\right)^{\omega_{j}}+\prod_{j=1}^{k} v_{\alpha_{\sigma(j)}}^{\omega_{j}}}\right)$.

Then, for $n=k+1$, according to Definition 6 and operational laws, we have:

$$
\begin{aligned}
& \operatorname{IFCA}_{\omega}^{\varepsilon}\left(\alpha_{1}, \alpha_{2}, \ldots, \alpha_{k+1}\right)=\left(\frac{\prod_{j=1}^{k}\left(1+\mu_{\alpha_{\sigma(j)}}\right)^{\omega_{j}}-\prod_{j=1}^{k}\left(1-\mu_{\alpha_{\sigma(j)}}\right)^{\omega_{j}}}{\prod_{j=1}^{k}\left(1+\mu_{\alpha_{\sigma(j)}}\right)^{\omega_{j}}+\prod_{j=1}^{k}\left(1-\mu_{\alpha_{\sigma(j)}}\right)^{\omega_{j}}}, \frac{2 \prod_{j=1}^{k} v_{\alpha_{\sigma(j)}}^{\omega_{j}}}{\prod_{j=1}^{k}\left(2-v_{\alpha_{\sigma(j)}}\right)^{\omega_{j}}+\prod_{j=1}^{k} v_{\alpha_{\sigma(j)}}^{\omega_{j}}}\right) \\
& \oplus_{\varepsilon}\left(\frac{\left(1+\mu_{\alpha_{\sigma(k+1)}}\right)^{\omega_{k+1}}-\left(1-\mu_{\alpha_{\sigma(k+1)}}\right)^{\omega_{k+1}}}{\left(1+\mu_{\alpha_{\sigma(k+1)}}\right)^{\omega_{k+1}}+\left(1-\mu_{\alpha_{\sigma(k+1)}}\right)^{w_{k+1}}}, \frac{2\left(v_{\alpha_{\sigma(k+1)}}\right)^{\omega_{k+1}}}{\left(2-v_{\alpha_{\sigma(k+1)}}\right)^{w_{k+1}}+\left(v_{\alpha_{\sigma(k+1)}}\right)^{\omega_{k+1}}}\right)= \\
& \left(\frac{\prod_{j=1}^{k+1}\left(1+\mu_{\alpha_{\sigma(j)}}\right)^{\omega_{j}}-\prod_{j=1}^{k+1}\left(1-\mu_{\alpha_{\sigma(j)}}\right)^{\omega_{j}}}{\prod_{j=1}^{k+1}\left(1+\mu_{\alpha_{\sigma(j)}}\right)^{\omega_{j}}+\prod_{j=1}^{k+1}\left(1-\mu_{\alpha_{\sigma(j)}}\right)^{\omega_{j}}}, \frac{2 \prod_{j=1}^{k+1} v_{\alpha_{\sigma(j)}}^{\omega_{j}}}{\prod_{j=1}^{k+1}\left(2-v_{\alpha_{\sigma(j)}}\right)^{\omega_{j}}+\prod_{j=1}^{k+1} v_{\alpha_{\sigma(j)}}^{\omega_{j}}}\right) .
\end{aligned}
$$


That is, for $n=k+1$, the Eq. (19) always holds, which completes the proof of Theorem 5 .

Now, we consider three special cases of the IFCA $^{\varepsilon}$ operator.

(1) If Eq. (8) holds, then:

$$
m\left(\left\{x_{\sigma(j)}\right\}\right)=m\left(A_{\sigma(j)}\right)-m\left(A_{\sigma(j-1)}\right), j=1,2, \ldots, n,
$$

in this case, the IFCA $^{\varepsilon}$ operator Eqs. (18), (19) reduce to the intuitionistic fuzzy Einstein weighted averaging $\left(\right.$ IFWA $\left.^{\varepsilon}\right)$ operator (Eq. (21)):

$$
\begin{aligned}
& \operatorname{IFWA}^{\varepsilon}\left(\alpha_{1}, \alpha_{2}, \ldots, \alpha_{n}\right)=\left(m\left(\left\{x_{1}\right\}\right)\right) \cdot_{\varepsilon} \alpha_{1} \oplus_{\varepsilon}\left(m\left(\left\{x_{2}\right\}\right)\right){ }_{\varepsilon} \alpha_{2} \oplus_{\varepsilon} \ldots \oplus_{\varepsilon}\left(m\left(\left\{x_{n}\right\}\right)\right){ }_{\varepsilon} \alpha_{n}= \\
& \left(\frac{\prod_{j=1}^{n}\left(1+\mu_{\alpha_{j}}\right)^{m\left(\left\{x_{j}\right\}\right)}-\prod_{j=1}^{n}\left(1-\mu_{\alpha_{j}}\right)^{m\left(\left\{x_{j}\right\}\right)}}{\prod_{j=1}^{n}\left(1+\mu_{\alpha_{j}}\right)^{m\left(\left\{x_{j}\right\}\right)}+\prod_{j=1}^{n}\left(1-\mu_{\alpha_{j}}\right)^{m\left(\left\{x_{j}\right\}\right)}}, \frac{2 \prod_{j=1}^{n} v_{\alpha_{j}}^{m\left(\left\{x_{j}\right\}\right)}}{\prod_{j=1}^{n}\left(2-v_{\alpha_{j}}\right)^{m\left(\left\{x_{j}\right\}\right)}+\prod_{j=1}^{n} v_{\alpha_{j}}^{m\left(\left\{x_{j}\right\}\right)}}\right) .
\end{aligned}
$$

In particular, if $m\left(\left\{x_{j}\right\}\right)=\frac{1}{n}$, for all $j=1,2, \ldots, n$, then IFWA ${ }^{\varepsilon}$ operator Eq. (21) reduces to the intuitionistic fuzzy Einstein averaging $\left(\operatorname{IFA}^{\varepsilon}\right)$ operator:

$$
\begin{aligned}
& \operatorname{IFA}^{\varepsilon}\left(\alpha_{1}, \alpha_{2}, \ldots, \alpha_{n}\right)=\left(\frac{1}{n}\right) \cdot{ }_{\varepsilon}\left(\alpha_{1} \oplus_{\varepsilon} \alpha_{2} \oplus_{\varepsilon} \cdots \oplus_{\varepsilon} \alpha_{n}\right)= \\
& \left(\frac{\prod_{j=1}^{n}\left(1+\mu_{\alpha_{j}}\right)^{1 / n}-\prod_{j=1}^{n}\left(1-\mu_{\alpha_{j}}\right)^{1 / n}}{\prod_{j=1}^{n}\left(1+\mu_{\alpha_{j}}\right)^{1 / n}+\prod_{j=1}^{n}\left(1-\mu_{\alpha_{j}}\right)^{1 / n}}, \frac{2 \prod_{j=1}^{n} v_{\alpha_{j}}^{1 / n}}{\prod_{j=1}^{n}\left(2-v_{\alpha_{j}}\right)^{1 / n}+\prod_{j=1}^{n} v_{\alpha_{j}}^{1 / n}}\right) .
\end{aligned}
$$

(2) If:

$$
m(A)=\sum_{j=1}^{A} w_{j}, \text { for all } A \subseteq X,
$$

where $|A|$ is the number of the elements in the set $A$, then:

$$
w_{j}=m\left(A_{\sigma(j)}\right)-m\left(A_{\sigma(j-1)}\right), j=1,2, \ldots, n,
$$

where: $w=\left(w_{1}, w_{2}, \ldots, w_{n}\right)^{T}, w_{j} \geq 0, j=1,2, \ldots, n$, and $\sum_{j=1}^{n} w_{j}=1$. In this case, the IFCA ${ }^{\varepsilon}$ operator Eqs. (18), (19), reduce to the intuitionistic fuzzy Einstein ordered weighted averaging $\left(\right.$ IFOWA $^{\varepsilon}$ ) operator (Eq. (25)):

$$
\begin{aligned}
& \operatorname{IFOWA}^{\varepsilon}\left(\alpha_{1}, \alpha_{2}, \ldots, \alpha_{n}\right)=\left(w_{1}\right) \cdot_{\varepsilon} \alpha_{\sigma(1)} \oplus_{\varepsilon}\left(w_{2}\right) \cdot_{\varepsilon} \alpha_{\sigma(2)} \oplus_{\varepsilon} \ldots \oplus_{\varepsilon}\left(w_{n}\right){ }_{\varepsilon} \alpha_{\sigma(n)}= \\
& \left(\frac{\prod_{j=1}^{n}\left(1+\mu_{\alpha_{\sigma(j)}}\right)^{w_{j}}-\prod_{j=1}^{n}\left(1-\mu_{\alpha_{\sigma(j)}}\right)^{w_{j}}}{\prod_{j=1}^{n}\left(1+\mu_{\alpha_{\sigma(j)}}\right)^{w_{j}}+\prod_{j=1}^{n}\left(1-\mu_{\alpha_{\sigma(j)}}\right)^{w_{j}}}, \frac{2 \prod_{j=1}^{n} v_{\alpha_{\sigma(j)}}^{w_{j}}}{\prod_{j=1}^{n}\left(2-v_{\alpha_{\sigma(j)}}\right)^{\omega_{j}}+\prod_{j=1}^{n} v_{\alpha_{\sigma(j)}}^{w_{j}}}\right) .
\end{aligned}
$$

In particular, if $m(A)=\frac{|A|}{n}$ for all $A \subseteq X$, then both the $\operatorname{IFCA}^{\varepsilon}$ operator (18) and IFOWA ${ }^{\varepsilon}$ operator (25) reduce to the IFA ${ }^{\varepsilon}$ operator (Eq. (22)).

(3) If:

$$
m(A)=Q\left(\sum_{x_{j} \in A} m\left(\left\{x_{j}\right\}\right)\right), \text { for all } A \subseteq X,
$$

where: $Q$ is a basic unit-interval monotonic (BUM) function $Q:[0,1] \rightarrow[0,1]$ and is monotonic with the following properties: (i) $Q(0)=0$; (ii) $Q(1)=1$; and (iii) $Q(x) \geq Q(y)$ for all $x>y$. Then we let: 


$$
\omega_{j}=m\left(A_{\sigma(j)}\right)-m\left(A_{\sigma(j-1)}\right)=Q\left(\sum_{i \leq j} m\left(\left\{x_{\sigma(j)}\right\}\right)\right)-Q\left(\sum_{i<j} m\left(\left\{x_{\sigma(j)}\right\}\right)\right), j=1,2, \ldots, n,
$$

where: $\omega=\left(\omega_{1}, \omega_{2}, \ldots, \omega_{n}\right)^{T}, \omega_{j} \geq 0, j=1,2, \ldots, n$, and $\sum_{j=1}^{n} \omega_{j}=1$. In this case, the IFCA ${ }^{\varepsilon}$ operator Eqs. (18), (19) reduce to the following form:

$$
\begin{gathered}
\operatorname{IFWOWA}^{\varepsilon}\left(\alpha_{1}, \alpha_{2}, \ldots, \alpha_{n}\right)=\left(\omega_{1}\right)_{\varepsilon} \alpha_{\sigma(1)} \oplus_{\varepsilon}\left(\omega_{2}\right)_{\varepsilon} \alpha_{\sigma(2)} \oplus_{\varepsilon} \ldots \oplus_{\varepsilon}\left(\omega_{n}\right) \cdot_{\varepsilon} \alpha_{\sigma(n)}= \\
\left(\frac{\prod_{j=1}^{n}\left(1+\mu_{\alpha_{\sigma(j)}}\right)^{\omega_{j}}-\prod_{j=1}^{n}\left(1-\mu_{\alpha_{\sigma(j)}}\right)^{\omega_{j}}}{\prod_{j=1}^{n}\left(1+\mu_{\alpha_{\sigma(j)}}\right)^{\omega_{j}}+\prod_{j=1}^{n}\left(1-\mu_{\alpha_{\sigma(j)}}\right)^{\omega_{j}}}, \frac{2 \prod_{j=1}^{n} v_{\alpha_{\sigma(j)}}^{\omega_{j}}}{\prod_{j=1}^{n}\left(2-v_{\alpha_{\sigma(j)}}\right)^{\omega_{j}}+\prod_{j=1}^{n} v_{\alpha_{\sigma(j)}}^{\omega_{j}}}\right),
\end{gathered}
$$

which we call an intuitionistic fuzzy Einstein weighted ordered weighted averaging (IFWOWA ${ }^{\varepsilon}$ ) operator. In particular, if $m\left(\left\{x_{j}\right\}\right)=\frac{1}{n}$, for all $j=1,2, \ldots, n$, then the IFWOWA ${ }^{\varepsilon}$ reduces to the $\mathrm{IFOWA}^{\varepsilon}$ operator.

In the following, let us look at some desirable properties of the IFCA $^{\varepsilon}$.

Theorem 4 (Idempotency). Let $\alpha_{j}=\left(\mu_{\alpha_{j}}, v_{\alpha_{j}}\right) \quad(j=1,2, \ldots, n)$ be a collection of IFVs, and $m$ be a fuzzy measure on $X$, if all $\alpha_{j}(j=1,2, \ldots, n)$ are equal, i.e., $\alpha_{j}=\alpha$, for all $j$, then:

$$
\operatorname{IFCA}_{m}^{\varepsilon}\left(\alpha_{1}, \alpha_{2}, \ldots, \alpha_{n}\right)=\alpha \text {. }
$$

Proof. According to Theorem 3, if for all $\alpha_{j}=\alpha$, then:

$$
\begin{aligned}
& \operatorname{IFCA}_{m}^{\varepsilon}\left(\alpha_{1}, \alpha_{2}, \ldots, \alpha_{n}\right)= \\
& \left(\frac{\left(1+\mu_{\alpha}\right)^{\sum_{j=1}^{n} m\left(A_{\sigma(j)}\right)-m\left(A_{\sigma(j-1)}\right)}-\left(1-\mu_{\alpha}\right)^{\sum_{j=1}^{n} m\left(A_{\sigma(j)}\right)-m\left(A_{\sigma(j-1)}\right)}}{\left(1+\mu_{\alpha}\right)^{\sum_{j=1}^{n} m\left(A_{\sigma(j)}\right)-m\left(A_{\sigma(j-1)}\right)}+\left(1-\mu_{\alpha}\right)^{\sum_{j=1}^{n} m\left(A_{\sigma(j)}\right)-m\left(A_{\sigma(j-1)}\right)}},\right.
\end{aligned}
$$

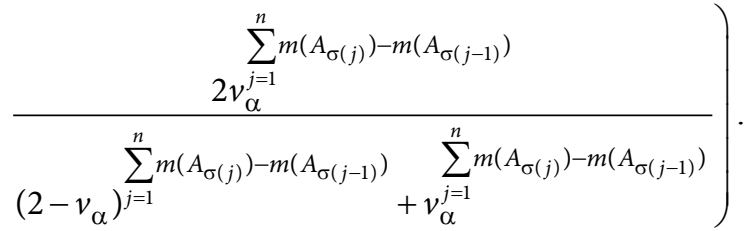

Since

$$
\begin{aligned}
& \sum_{j=1}^{n}\left(m\left(A_{\sigma(j)}\right)-m\left(A_{\sigma(j-1)}\right)\right)=\left(m\left(A_{\sigma(1)}\right)-m\left(A_{\sigma(0)}\right)\right)+\left(m\left(A_{\sigma(2)}\right)-m\left(A_{\sigma(1)}\right)\right) \\
& +\ldots+\left(m\left(A_{\sigma(n)}\right)-m\left(A_{\sigma(n-1)}\right)\right)= \\
& m\left(A_{\sigma(n)}\right)-m\left(A_{\sigma(0)}\right)=1 .
\end{aligned}
$$

Thus,

$$
\operatorname{IFCA}_{m}^{\varepsilon}\left(\alpha_{1}, \alpha_{2}, \ldots, \alpha_{n}\right)=\left(\mu_{\alpha}, v_{\alpha}\right)=\alpha
$$


Theorem 5 (Boundary). Let $\alpha_{j}=\left(\mu_{\alpha_{j}}, v_{\alpha_{j}}\right)(j=1,2, \ldots, n)$ be a collection of IFVs, and $m$ be a fuzzy measure on $X$, and let $\alpha^{-}=\left(\min _{j}\left(\mu_{\alpha_{j}}\right), \max _{j}\left(v_{\alpha_{j}}\right)\right), \alpha^{+}=\left(\max _{j}\left(\mu_{\alpha_{j}}\right), \min _{j}\left(v_{\alpha_{j}}\right)\right)$, then:

$$
\alpha^{-} \leq \operatorname{IFCA}_{m}^{\varepsilon}\left(\alpha_{1}, \alpha_{2}, \ldots, \alpha_{n}\right) \leq \alpha^{+} \text {. }
$$

Proof. Since $\min _{j}\left(\mu_{\alpha_{j}}\right) \leq \mu_{\alpha_{\sigma(j)}} \leq \max _{j}\left(\mu_{\alpha_{j}}\right)$, for all $j$, then:

$$
\begin{aligned}
\min _{j}\left(\mu_{\alpha_{j}}\right)= & \frac{\prod_{j=1}^{n}\left(1+\min _{j}\left(\mu_{\alpha_{j}}\right)\right)^{w_{j}}-\prod_{j=1}^{n}\left(1-\min _{j}\left(\mu_{\alpha_{j}}\right)\right)^{w_{j}}}{\prod_{j=1}^{n}\left(1+\min _{j}\left(\mu_{\alpha_{j}}\right)\right)^{w_{j}}+\prod_{j=1}^{n}\left(1-\min _{j}\left(\mu_{\alpha_{j}}\right)\right)^{w_{j}}} \leq \\
& \frac{\prod_{j=1}^{n}\left(1+\mu_{\alpha_{\sigma(j)}}\right)^{w_{j}}-\prod_{j=1}^{n}\left(1-\mu_{\alpha_{\sigma(j)}}\right)^{w_{j}}}{\prod_{j=1}^{n}\left(1+\mu_{\alpha_{\sigma(j)}}\right)^{w_{j}}+\prod_{j=1}^{n}\left(1-\mu_{\alpha_{\sigma(j)}}\right)^{w_{j}}} \leq \\
& \frac{\prod_{j=1}^{n}\left(1+\max _{j}\left(\mu_{\alpha_{j}}\right)\right)^{w_{j}}-\prod_{j=1}^{n}\left(1-\max _{j}\left(\mu_{\alpha_{j}}\right)\right)^{w_{j}}}{\prod_{j=1}^{n}\left(1+\max _{j}\left(\mu_{\alpha_{j}}\right)\right)^{w_{j}}+\prod_{j=1}^{n}\left(1-\max _{j}\left(\mu_{\alpha_{j}}\right)\right)^{w_{j}}}=\max _{j}\left(\mu_{\alpha_{j}}\right),
\end{aligned}
$$

i.e.:

$$
\min _{j}\left(\mu_{\alpha_{j}}\right) \leq \frac{\prod_{j=1}^{n}\left(1+\mu_{\alpha_{\sigma(j)}}\right)^{w_{j}}-\prod_{j=1}^{n}\left(1-\mu_{\alpha_{\sigma(j)}}\right)^{w_{j}}}{\prod_{j=1}^{n}\left(1+\mu_{\alpha_{\sigma(j)}}\right)^{w_{j}}+\prod_{j=1}^{n}\left(1-\mu_{\alpha_{\sigma(j)}}\right)^{w_{j}}} \leq \max _{j}\left(\mu_{\alpha_{j}}\right) .
$$

Similarly, since $\min _{j}\left(v_{\alpha_{j}}\right) \leq v_{\alpha_{\sigma(j)}} \leq \max _{j}\left(v_{\alpha_{j}}\right)$, then:

$$
\begin{aligned}
& \min _{j}\left(v_{\alpha_{j}}\right)=\frac{2 \prod_{j=1}^{n}\left(\min _{j}\left(v_{\alpha_{j}}\right)\right)^{w_{j}}}{\prod_{j=1}^{n}\left(2-\min _{j}\left(v_{\alpha_{j}}\right)\right)^{w_{j}}+\prod_{j=1}^{n}\left(\min _{j}\left(v_{\alpha_{j}}\right)\right)^{w_{j}}} \leq \frac{2 \prod_{j=1}^{n} v_{\alpha_{\sigma(j)}}^{w_{j}}}{\prod_{j=1}^{n}\left(2-v_{\alpha_{\sigma(j)}}\right)^{w_{j}}+\prod_{j=1}^{n} v_{\alpha_{\sigma(j)}}^{w_{j}}} \leq \\
& \frac{2 \prod_{j=1}^{n}\left(\max _{j}\left(v_{\alpha_{j}}\right)\right)^{w_{j}}}{\prod_{j=1}^{n}\left(2-\max _{j}\left(v_{\alpha_{j}}\right)\right)^{w_{j}}+\prod_{j=1}^{n}\left(\max _{j}\left(v_{\alpha_{j}}\right)\right)^{w_{j}}}=\max _{j}\left(v_{\alpha_{j}}\right),
\end{aligned}
$$

i.e.:

$$
\min _{j}\left(v_{\alpha_{j}}\right) \leq \frac{2 \prod_{j=1}^{n} v_{\alpha_{\sigma(j)}}^{w_{j}}}{\prod_{j=1}^{n}\left(2-v_{\alpha_{\sigma(j)}}\right)^{w_{j}}+\prod_{j=1}^{n} v_{\alpha_{\sigma(j)}}^{w_{j}}} \leq \max _{j}\left(v_{\alpha_{j}}\right) .
$$

From the Definition 1 and Eqs. (31) and (32), we have:

$$
\alpha^{-} \leq \operatorname{IFCA}_{m}^{\varepsilon}\left(\alpha_{1}, \alpha_{2}, \ldots, \alpha_{n}\right) \leq \alpha^{+} .
$$


Theorem 6 (Monotonicity). Let $\alpha_{j}=\left(\mu_{\alpha_{j}}, v_{\alpha_{j}}\right)(j=1,2, \ldots, n)$ be a collection of IFVs, and $m$ be a fuzzy measure on $X$, if $\mu_{\beta_{\sigma(j)}} \leq \mu_{\alpha_{\sigma(j)}}, v_{\beta_{\sigma(j)}} \geq v_{\alpha_{\sigma(j)}}$, for all $j$, then:

$$
\operatorname{IFCA}_{m}^{\varepsilon}\left(\beta_{1}, \beta_{2}, \ldots, \beta_{n}\right) \leq \operatorname{IFCA}_{m}^{\varepsilon}\left(\alpha_{1}, \alpha_{2}, \ldots, \alpha_{n}\right) .
$$

Proof. It is straightforward and thus omitted.

Theorem 7 (Commutativity). Let $\alpha_{j}=\left(\mu_{\alpha_{j}}, v_{\alpha_{j}}\right), \alpha_{j}^{\prime}=\left(\mu_{\alpha_{j}^{\prime}}, v_{\alpha_{j}^{\prime}}\right)(j=1,2, \ldots, n)$ be two collections of IFVs, and $m$ be a fuzzy measure on $X$, then:

$$
\operatorname{IFCA}_{m}^{\varepsilon}\left(\alpha_{1}^{\prime}, \alpha_{2}^{\prime}, \ldots, \alpha_{n}^{\prime}\right)=\operatorname{IFCA}_{m}^{\varepsilon}\left(\alpha_{1}, \alpha_{2}, \ldots, \alpha_{n}\right),
$$

where $\left(\alpha_{1}^{\prime}, \alpha_{2}^{\prime}, \ldots, \alpha_{n}^{\prime}\right)$ is any permutation of $\left(\alpha_{1}, \alpha_{2}, \ldots, \alpha_{n}\right)$.

In the following, we analyze the relationship between the IFCA ${ }^{\varepsilon}$ operator and IFCA operator proposed by $\mathrm{Xu}$ (2010), we first introduce the following lemma.

Lemma $1(\mathrm{Xu}, \mathrm{Da} 2002)$. Let $x_{j}>0, \lambda_{j}>0, j=1,2, \ldots, n$, and $\sum_{j=1}^{n} \lambda_{j}=1$, then:

$$
\prod_{j=1}^{n} x_{j}^{\lambda_{j}} \leq \sum_{j=1}^{n} \lambda_{j} x_{j},
$$

with equality if and only if $x_{1}=x_{2}=\ldots=x_{n}$.

Theorem 8. Let $\alpha_{j}=\left(\mu_{\alpha_{j}}, v_{\alpha_{j}}\right)(j=1,2, \ldots, n)$ be a collection of IFVs, and $m$ be a fuzzy measure on $X$, then:

$$
\operatorname{IFCA}_{m}\left(\alpha_{1}, \alpha_{2}, \ldots, \alpha_{n}\right) \geq \operatorname{IFCA}_{m}^{\varepsilon}\left(\alpha_{1}, \alpha_{2}, \ldots, \alpha_{n}\right) .
$$

Proof. Since $\prod_{j=1}^{n}\left(1+\mu_{\alpha}\right)^{m\left(A_{\sigma(j)}\right)-m\left(A_{\sigma(j-1)}\right)}+\prod_{j=1}^{n}\left(1-\mu_{\alpha}\right)^{m\left(A_{\sigma(j)}\right)-m\left(A_{\sigma(j-1)}\right)} \leq$

$\sum_{j=1}^{n}\left(m\left(A_{\sigma(j)}\right)-m\left(A_{\sigma(j-1)}\right)\right)\left(1+\mu_{\alpha_{j}}\right)+\sum_{j=1}^{n}\left(m\left(A_{\sigma(j)}\right)-m\left(A_{\sigma(j-1)}\right)\right)\left(1-\mu_{\alpha_{j}}\right)=2$,

then:

$$
\begin{aligned}
& \frac{\prod_{j=1}^{n}\left(1+\mu_{\alpha_{\sigma(j)}}\right)^{m\left(A_{\sigma(j)}\right)-m\left(A_{\sigma(j-1)}\right)}-\prod_{j=1}^{n}\left(1-\mu_{\alpha_{\sigma(j)}}\right)^{m\left(A_{\sigma(j)}\right)-m\left(A_{\sigma(j-1)}\right)}}{\prod_{j=1}^{n}\left(1+\mu_{\alpha_{\sigma(j)}}\right)^{m\left(A_{\sigma(j)}\right)-m\left(A_{\sigma(j-1)}\right)}+\prod_{j=1}^{n}\left(1-\mu_{\alpha_{\sigma(j)}}\right)^{m\left(A_{\sigma(j)}\right)-m\left(A_{\sigma(j-1)}\right)}}= \\
& 1-\frac{2 \prod_{j=1}^{n}\left(1-\mu_{\alpha_{\sigma(j)}}\right)^{m\left(A_{\sigma(j)}\right)-m\left(A_{\sigma(j-1)}\right)}}{\prod_{j=1}^{n}\left(1+\mu_{\alpha_{\sigma(j)}}\right)^{m\left(A_{\sigma(j)}\right)-m\left(A_{\sigma(j-1)}\right)}+\prod_{j=1}^{n}\left(1-\mu_{\alpha_{\sigma(j)}}\right)^{m\left(A_{\sigma(j)}\right)-m\left(A_{\sigma(j-1)}\right)}} \leq \\
& 1-\prod_{j=1}^{n}\left(1-\mu_{\alpha_{\sigma(j)}}\right)^{m\left(A_{\sigma(j)}\right)-m\left(A_{\sigma(j-1)}\right)},
\end{aligned}
$$

where the equality holds if and only if $\mu_{\alpha_{\sigma(j)}}(j=1,2, \ldots, n)$ are equal.

$$
\begin{aligned}
& \text { Also, since } \prod_{j=1}^{n}\left(2-v_{\alpha_{\sigma(j)}}\right)^{m\left(A_{\sigma(j)}\right)-m\left(A_{\sigma(j-1)}\right)}+\prod_{j=1}^{n} v_{\alpha_{\sigma(j)}}^{m\left(A_{\sigma(j)}\right)-m\left(A_{\sigma(j-1)}\right)} \leq \\
& \qquad \sum_{j=1}^{n}\left(m\left(A_{\sigma(j)}\right)-m\left(A_{\sigma(j-1)}\right)\right)\left(2-v_{\alpha_{\sigma(j)}}\right)+\sum_{j=1}^{n}\left(m\left(A_{\sigma(j)}\right)-m\left(A_{\sigma(j-1)}\right)\right) v_{\alpha_{\sigma(j)}}=2,
\end{aligned}
$$

then:

$$
\frac{2 \prod_{j=1}^{n} v_{\alpha_{\sigma(j)}}^{m\left(A_{\sigma(j)}\right)-m\left(A_{\sigma(j-1)}\right)}}{\prod_{j=1}^{n}\left(2-v_{\alpha_{\sigma(j)}}\right)^{m\left(A_{\sigma(j)}\right)-m\left(A_{\sigma(j-1)}\right)}+\prod_{j=1}^{n} v_{\alpha_{\sigma(j)}}^{m\left(A_{\sigma(j)}\right)-m\left(A_{\sigma(j-1)}\right)}} \geq \prod_{j=1}^{n} v_{\alpha_{\sigma(j)}}^{m\left(A_{\sigma(j)}\right)-m\left(A_{\sigma(j-1)}\right)} .
$$


Let IFCA $\operatorname{Im}_{m}\left(\alpha_{1}, \alpha_{2}, \ldots, \alpha_{n}\right)=\alpha=\left(\mu_{\alpha}, v_{\alpha}\right), \operatorname{IFCA}_{m}^{\varepsilon}\left(\alpha_{1}, \alpha_{2}, \ldots, \alpha_{n}\right)=\alpha^{\varepsilon}=\left(\mu_{\alpha}^{\varepsilon}, v_{\alpha}^{\varepsilon}\right)$, then Eqs. (36) and (37) are transformed into the forms $\mu_{\alpha} \geq \mu_{\alpha}^{\varepsilon}$ and $v_{\alpha} \leq v_{\alpha}^{\varepsilon}$, respectively. Thus, $S(\alpha)=\mu_{\alpha}-v_{\alpha} \geq \mu_{\alpha}^{\varepsilon}-v_{\alpha}^{\varepsilon}=s\left(\alpha^{\varepsilon}\right)$, by Definition 1, we have:

$$
\operatorname{IFCA}_{m}\left(\alpha_{1}, \alpha_{2}, \ldots, \alpha_{n}\right) \geq \operatorname{IFCA}_{m}^{\varepsilon}\left(\alpha_{1}, \alpha_{2}, \ldots, \alpha_{n}\right)
$$

which completes the proof of Theorem 11 .

Example 1. Let $m$ be a fuzzy measure on $P(X), X=\left\{x_{1}, x_{2}, x_{3}, x_{4}\right\}$ in which:

$$
\begin{aligned}
& m(\varnothing)=0, m\left(\left\{x_{1}\right\}\right)=m\left(\left\{x_{2}\right\}\right)=0.3, m\left(\left\{x_{3}\right\}\right)=0.4, m\left(\left\{x_{4}\right\}\right)=0.1, \\
& m\left(\left\{x_{1}, x_{2}\right\}\right)=0.6, m\left(\left\{x_{1}, x_{3}\right\}\right)=0.5, m\left(\left\{x_{1}, x_{4}\right\}\right)=0.4, m\left(\left\{x_{2}, x_{3}\right\}\right)=0.5, \\
& m\left(\left\{x_{2}, x_{4}\right\}\right)=0.5, m\left(\left\{x_{3}, x_{4}\right\}\right)=0.6, m\left(\left\{x_{1}, x_{2}, x_{3}\right\}\right)=0.7, m\left(\left\{x_{1}, x_{2}, x_{4}\right\}\right)=0.8, \\
& m\left(\left\{x_{1}, x_{3}, x_{4}\right\}\right)=0.7, m\left(\left\{x_{2}, x_{3}, x_{4}\right\}\right)=0.9, m\left(\left\{x_{1}, x_{2}, x_{3}, x_{4}\right\}\right)=1 .
\end{aligned}
$$

Let $\alpha_{1}=(0.2,0.5), \alpha_{2}=(0.4,0.2), \alpha_{3}=(0.5,0.4)$, and $\alpha_{4}=(0.7,0.1)$ be five IFVs on $X=\left\{x_{1}, x_{2}, x_{3}, x_{4}\right\}$, respectively, then we arrange the IFVs in descending order by Definition 1, we have:

$$
\alpha_{\sigma(1)}=\alpha_{4}=(0.7,0.1), \alpha_{\sigma(2)}=\alpha_{2}=(0.4,0.2), \alpha_{\sigma(3)}=\alpha_{3}=(0.5,0.4), \alpha_{\sigma(4)}=\alpha_{1}=(0.2,0.5) .
$$

Then:

$$
A_{\sigma(1)}=\left\{x_{4}\right\}, A_{\sigma(2)}=\left\{x_{2}, x_{4}\right\}, A_{\sigma(3)}=\left\{x_{2}, x_{3}, x_{4}\right\}, A_{\sigma(4)}=\left\{x_{1}, x_{2}, x_{3}, x_{4}\right\},
$$

with the IFCA ${ }^{\varepsilon}$ operator Eq. (19), we have:

$$
\operatorname{IFCA}_{m}^{\varepsilon}\left(\alpha_{1}, \alpha_{2}, \alpha_{3}, \alpha_{4}\right)=(0.4591,0.2747) \text {. }
$$

If we use the IFC operator (i.e. Eq. (12)) to aggregate four IFVs, then we have:

$\operatorname{IFCA}_{m}\left(\alpha_{1}, \alpha_{2}, \alpha_{3}, \alpha_{4}\right)=(0.5363,0.2699)$.

Obviously, we have $\operatorname{IFCA}_{m}\left(\alpha_{1}, \alpha_{2}, \alpha_{3}, \alpha_{4}\right) \geq \operatorname{IFCA}_{m}^{\varepsilon}\left(\alpha_{1}, \alpha_{2}, \alpha_{3}, \alpha_{4}\right)$.

\subsection{Intuitionistic fuzzy Einstein Choquet geometric operator}

Definition 8. Let $\alpha_{j}=\left(\mu_{\alpha_{j}}, v_{\alpha_{j}}\right) \quad(j=1,2, \ldots, n)$ be a collection of IFVs on $X$, and $m$ be a fuzzy measure on $X$, then we call

$$
\begin{aligned}
& \operatorname{IFCG}_{m}^{\varepsilon}\left(\alpha_{1}, \alpha_{2}, \ldots, \alpha_{n}\right)=\left(\alpha_{\sigma(1)}\right)^{\wedge_{\varepsilon}\left(m\left(A_{\sigma(1)}\right)-m\left(A_{\sigma(0)}\right)\right)} \otimes_{\varepsilon}\left(\alpha_{\sigma(2)}\right)^{\wedge_{\varepsilon}\left(m\left(A_{\sigma(2)}\right)-m\left(A_{\sigma(1)}\right)\right)} \\
& \otimes_{\varepsilon} \ldots \otimes_{\varepsilon}\left(\alpha_{\sigma(n)}\right)^{\wedge_{\varepsilon}\left(m\left(A_{\sigma(n)}\right)-m\left(A_{\sigma(n-1)}\right)\right)}
\end{aligned}
$$

an intuitionistic fuzzy Einstein Choquet geometric (IFCG ${ }^{\varepsilon}$ ) operator, where $(\sigma(1), \sigma(2), \ldots, \sigma(n))$ is a permutation of $(1,2, \ldots, n)$ such that $\alpha_{\sigma(j-1)} \geq \alpha_{\sigma(j)}$ for all $j=2,3, \ldots, n$, $A_{\sigma(j)}=\left\{x_{\sigma(1)}, x_{\sigma(2)}, \ldots, x_{\sigma(j)}\right\}$ and $A_{\sigma(0)}=\varnothing$. 
Theorem 9. Let $\alpha_{j}=\left(\mu_{\alpha_{j}}, v_{\alpha_{j}}\right)(j=1,2, \ldots, n)$ be a collection of IFVs on $X$, and $m$ be a fuzzy measure on $X$, then their aggregated value by using IFCG $^{\varepsilon}$ operator is also an IFV, and $\operatorname{IFCG}_{m}^{\varepsilon}\left(\alpha_{1}, \alpha_{2}, \ldots, \alpha_{n}\right)=$

$$
\left.\begin{array}{l}
\left(\frac{2 \prod_{j=1}^{n} \mu_{\alpha_{\sigma(j)}}^{m\left(A_{\sigma(j)}\right)-m\left(A_{\sigma(j-1)}\right)}}{\prod_{j=1}^{n}\left(2-\mu_{\alpha_{\sigma(j)}}\right)^{m\left(A_{\sigma(j)}\right)-m\left(A_{\sigma(j-1)}\right)}+\prod_{j=1}^{n} \mu_{\alpha_{\sigma(j)}}^{m\left(A_{\sigma(j)}\right)-m\left(A_{\sigma(j-1)}\right)}},\right. \\
\prod_{j=1}^{n}\left(1+v_{\alpha_{\sigma(j)}}\right)^{m\left(A_{\sigma(j)}\right)-m\left(A_{\sigma(j-1)}\right)}-\prod_{j=1}^{n}\left(1-v_{\alpha_{\sigma(j)}}\right)^{m\left(A_{\sigma(j)}\right)-m\left(A_{\sigma(j-1)}\right)} \\
\prod_{j=1}^{n}\left(1+v_{\alpha_{\sigma(j)}}\right)^{m\left(A_{\sigma(j)}\right)-m\left(A_{\sigma(j-1)}\right)}+\prod_{j=1}^{n}\left(1-v_{\alpha_{\sigma(j)}}\right)^{m\left(A_{\sigma(j)}\right)-m\left(A_{\sigma(j-1)}\right)}
\end{array}\right) .
$$

We will now consider three special cases of the $\mathrm{IFCG}^{\varepsilon}$ operator.

(1) If Eqs. (8) and (20) hold, then the IFCG ${ }^{\varepsilon}$ operator Eqs. (39), (40) reduce to the intuitionistic fuzzy Einstein weighted geometric $\left(\mathrm{IFWG}^{\varepsilon}\right.$ ) (Wang, Liu 2011) operator:

$$
\begin{aligned}
& \operatorname{IFWG}^{\varepsilon}\left(\alpha_{1}, \alpha_{2}, \ldots, \alpha_{n}\right)=\left(\alpha_{1}\right)^{\wedge_{\varepsilon}\left(m\left(\left\{x_{1}\right\}\right)\right)} \otimes_{\varepsilon}\left(\alpha_{2}\right)^{\wedge_{\varepsilon}\left(m\left(\left\{x_{2}\right\}\right)\right)} \otimes_{\varepsilon} \ldots \otimes_{\varepsilon}\left(\alpha_{n}\right)^{\wedge_{\varepsilon}\left(m\left(\left\{x_{n}\right\}\right)\right)}= \\
& \left(\frac{2 \prod_{j=1}^{n} \mu_{\alpha_{j}}^{m\left(\left\{x_{j}\right\}\right)}}{\prod_{j=1}^{n}\left(2-\mu_{\alpha_{j}}\right)^{m\left(\left\{x_{j}\right\}\right)}+\prod_{j=1}^{n} \mu_{\alpha_{j}}^{m\left(\left\{x_{j}\right\}\right)}}, \frac{\prod_{j=1}^{n}\left(1+v_{\alpha_{j}}\right)^{m\left(\left\{x_{j}\right\}\right)}-\prod_{j=1}^{n}\left(1-v_{\alpha_{j}}\right)^{m\left(\left\{x_{j}\right\}\right)}}{\prod_{j=1}^{n}\left(1+v_{\alpha_{j}}\right)^{m\left(\left\{x_{j}\right\}\right)}+\prod_{j=1}^{n}\left(1-v_{\alpha_{j}}\right)^{m\left(\left\{x_{j}\right\}\right)}}\right) \cdot
\end{aligned}
$$

In particular, if $m\left(\left\{x_{j}\right\}\right)=\frac{1}{n}$, for all $j=1,2, \ldots, n$, then IFWG $^{\varepsilon}$ operator Eq. (21) reduces to the intuitionistic fuzzy Einstein geometric averaging $\left(\mathrm{IFGA}^{\varepsilon}\right)$ operator:

$$
\begin{aligned}
& \operatorname{IFGA}^{\varepsilon}\left(\alpha_{1}, \alpha_{2}, \ldots, \alpha_{n}\right)=\left(\alpha_{1} \otimes_{\varepsilon} \alpha_{2} \otimes_{\varepsilon} \ldots \otimes_{\varepsilon} \alpha_{n}\right)^{\wedge} \frac{1}{n}= \\
& \left(\frac{2 \prod_{j=1}^{n} \mu_{\alpha_{j}}^{1 / n}}{\prod_{j=1}^{n}\left(2-\mu_{\alpha_{j}}\right)^{1 / n}+\prod_{j=1}^{n} \mu_{\alpha_{j}}^{1 / n}}, \frac{\prod_{j=1}^{n}\left(1+v_{\alpha_{j}}\right)^{1 / n}-\prod_{j=1}^{n}\left(1-v_{\alpha_{j}}\right)^{1 / n}}{\prod_{j=1}^{n}\left(1+v_{\alpha_{j}}\right)^{1 / n}+\prod_{j=1}^{n}\left(1-v_{\alpha_{j}}\right)^{1 / n}}\right) .
\end{aligned}
$$

(2) If Eqs. (23) and (24) hold, then the IFCG ${ }^{\varepsilon}$ operator Eqs. (39), (40) reduce to the intuitionistic fuzzy Einstein ordered weighted geometric (IFOWG ${ }^{\varepsilon}$ ) (Wang, Liu 2011) operator:

$$
\begin{aligned}
& \operatorname{IFOWG}^{\varepsilon}\left(\alpha_{1}, \alpha_{2}, \ldots, \alpha_{n}\right)=\left(\alpha_{\sigma(1)}\right)^{\wedge_{\varepsilon} w_{1}} \oplus_{\varepsilon}\left(\alpha_{\sigma(2)}\right)^{\wedge_{\varepsilon} w_{2}} \oplus_{\varepsilon} \cdots \oplus_{\varepsilon}\left(\alpha_{\sigma(n)}\right)^{\wedge_{\varepsilon} w_{n}}= \\
& \left(\frac{2 \prod_{j=1}^{n} \mu_{\alpha_{\sigma(j)}}^{w_{j}}}{\prod_{j=1}^{n}\left(2-\mu_{\alpha_{\sigma(j)}}\right)^{\omega_{j}}+\prod_{j=1}^{n} \mu_{\alpha_{\sigma(j)}}^{w_{j}}}, \frac{\prod_{j=1}^{n}\left(1+v_{\alpha_{\sigma(j)}}\right)^{w_{j}}-\prod_{j=1}^{n}\left(1-v_{\alpha_{\sigma(j)}}\right)^{w_{j}}}{\prod_{j=1}^{n}\left(1+v_{\alpha_{\sigma(j)}}\right)^{w_{j}}+\prod_{j=1}^{n}\left(1-v_{\alpha_{\sigma(j)}}\right)^{w_{j}}}\right) .
\end{aligned}
$$


In particular, if $m(A)=\frac{|A|}{n}$ for all $A \subseteq X$, then both the IFCG $^{\varepsilon}$ operator (18) and IFOWG ${ }^{\varepsilon}$ operator (25) reduce to the $\mathrm{IFGA}^{\varepsilon}$ operator.

(3) If Eqs. (26) and (27) hold, then the IFCG $^{\varepsilon}$ operator Eqs. (39), (40) reduce to the following form:

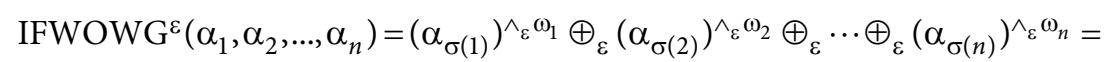

$$
\begin{aligned}
& \left(\frac{2 \prod_{j=1}^{n} \mu_{\alpha_{\sigma(j)}}^{\omega_{j}}}{\prod_{j=1}^{n}\left(2-\mu_{\alpha_{\sigma(j)}}\right)^{\omega_{j}}+\prod_{j=1}^{n} \mu_{\alpha_{\sigma(j)}}^{\omega_{j}}}, \frac{\prod_{j=1}^{n}\left(1+v_{\alpha_{\sigma(j)}}\right)^{\omega_{j}}-\prod_{j=1}^{n}\left(1-v_{\alpha_{\sigma(j)}}\right)^{\omega_{j}}}{\prod_{j=1}^{n}\left(1+v_{\alpha_{\sigma(j)}}\right)^{\omega_{j}}+\prod_{j=1}^{n}\left(1-v_{\alpha_{\sigma(j)}}\right)^{\omega_{j}}}\right),
\end{aligned}
$$

which we call an intuitionistic fuzzy Einstein weighted ordered weighted geometric (IFWOWG ${ }^{\varepsilon}$ ) operator. In particular, if $m\left(\left\{x_{j}\right\}\right)=\frac{1}{n}$, for all $j=1,2, \ldots, n$, then the IFWOWG ${ }^{\varepsilon}$ reduces to the $\mathrm{IFOWG}^{\varepsilon}$ operator.

In the following, let us look at some desirable properties of the IFCG $^{\varepsilon}$.

Theorem 10 (Idempotency). Let $\alpha_{j}=\left(\mu_{\alpha_{j}}, v_{\alpha_{j}}\right)(j=1,2, \ldots, n)$ be a collection of IFVs, and $m$ be a fuzzy measure on $X$, if all $\alpha_{j}(j=1,2, \ldots, n)$ are equal, i.e. $\alpha_{j}=\alpha$, for all $j$, then:

$$
\operatorname{IFCG}_{m}^{\varepsilon}\left(\alpha_{1}, \alpha_{2}, \ldots, \alpha_{n}\right)=\alpha
$$

Theorem 11 (Boundary). Let $\alpha_{j}=\left(\mu_{\alpha_{j}}, v_{\alpha_{j}}\right)(j=1,2, \ldots, n)$ be a collection of IFVs, and $m$ be a fuzzy measure on $X$, and let $\alpha^{-}=\left(\min _{j}\left(\mu_{\alpha_{j}}\right), \max _{j}\left(v_{\alpha_{j}}\right)\right), \alpha^{+}=\left(\max _{j}\left(\mu_{\alpha_{j}}\right), \min _{j}\left(v_{\alpha_{j}}\right)\right)$,
then:

$$
\alpha^{-} \leq \operatorname{IFCG}_{m}^{\varepsilon}\left(\alpha_{1}, \alpha_{2}, \ldots, \alpha_{n}\right) \leq \alpha^{+} .
$$

Theorem 12 (Monotonicity). Let $\alpha_{j}=\left(\mu_{\alpha_{j}}, v_{\alpha_{j}}\right)(j=1,2, \ldots, n)$ be a collection of IFVs, and $m$ be a fuzzy measure on $X$, if $\mu_{\beta_{\sigma(j)}} \leq \mu_{\alpha_{\sigma(j)}}, v_{\beta_{\sigma(j)}} \geq v_{\alpha_{\sigma(j)}}$, for all $j$, then:

$$
\operatorname{IFCG}_{m}^{\varepsilon}\left(\beta_{1}, \beta_{2}, \ldots, \beta_{n}\right) \leq \operatorname{IFCG}_{m}^{\varepsilon}\left(\alpha_{1}, \alpha_{2}, \ldots, \alpha_{n}\right)
$$

Proof. It is straightforward and thus omitted.

Theorem 13 (Commutativity). Let $\alpha_{j}=\left(\mu_{\alpha_{j}}, v_{\alpha_{j}}\right), \alpha_{j}^{\prime}=\left(\mu_{\alpha_{j}^{\prime}}, v_{\alpha_{j}^{\prime}}\right)(j=1,2, \ldots, n)$ be two collections of IFVs, and $m$ be a fuzzy measure on $X$, then:

$$
\operatorname{IFCG}_{m}^{\varepsilon}\left(\alpha_{1}^{\prime}, \alpha_{2}^{\prime}, \ldots, \alpha_{n}^{\prime}\right)=\operatorname{IFCG}_{m}^{\varepsilon}\left(\alpha_{1}, \alpha_{2}, \ldots, \alpha_{n}\right),
$$

where $\left(\alpha_{1}^{\prime}, \alpha_{2}^{\prime}, \ldots, \alpha_{n}^{\prime}\right)$ is any permutation of $\left(\alpha_{1}, \alpha_{2}, \ldots, \alpha_{n}\right)$.

In the following, we analyze the relationship between the IFCG $^{\varepsilon}$ operator and IFCG operator proposed by $\mathrm{Xu}(2010)$.

Theorem 14. Let $\alpha_{j}=\left(\mu_{\alpha_{j}}, v_{\alpha_{j}}\right) \quad(j=1,2, \ldots, n)$ be a collection of IFVs, and $m$ be a fuzzy measure on $X$, then:

$$
\operatorname{IFCG}_{m}\left(\alpha_{1}, \alpha_{2}, \ldots, \alpha_{n}\right) \leq \operatorname{IFCG}_{m}^{\varepsilon}\left(\alpha_{1}, \alpha_{2}, \ldots, \alpha_{n}\right) .
$$


Example 2. In Example 1, if we use IFCG $^{\varepsilon}$ operator and IFCG operator to aggregate the intuitionistic fuzzy information, we have:

$$
\begin{aligned}
& \operatorname{IFCG}_{m}^{\varepsilon}\left(\alpha_{1}, \alpha_{2}, \alpha_{3}, \alpha_{4}\right)=(0.4366,0.4591) ; \\
& \operatorname{IFCG}_{m}\left(\alpha_{1}, \alpha_{2}, \alpha_{3}, \alpha_{4}\right)=(0.4315,0.4644) .
\end{aligned}
$$

According to Definition 1, we have:

$\operatorname{IFCG}_{m}\left(\alpha_{1}, \alpha_{2}, \alpha_{3}, \alpha_{4}\right) \leq \operatorname{IFCG}_{m}^{\varepsilon}\left(\alpha_{1}, \alpha_{2}, \alpha_{3}, \alpha_{4}\right)$.

\section{An approach to decision making with intuitionistic fuzzy Einstein Choquet integral operators}

Let $A=\left\{a_{1}, a_{2}, \ldots, a_{m}\right\}$ be a finite set of alternatives, let $C=\left\{c_{1}, c_{2}, \ldots, c_{n}\right\}$ be a feasible set of attributes, let $R=\left(r_{i j}\right)_{m \times n}$ be an intuitionistic fuzzy decision matrix, where $r_{i j}=\left(\mu_{i j}, v_{i j}\right)$ is an IFV, provided by the decision maker for the alternative $a_{i} \in A$ with respect to the attribute $c_{j} \in C, \mu_{i j}$ indicates the degree the alternative $a_{i}$ should satisfy the attribute $c_{j}$ expressed by the decision maker, while $v_{i j}$ indicates the degree that the alternative $a_{i}$ should not satisfy the attribute $c_{j}$ expressed by the decision maker, and $\mu_{i j} \in[0,1], v_{i j} \in[0,1], \mu_{i j}+v_{i j} \leq 1$, $i=1,2, \ldots, m, j=1,2, \ldots ., n$.

In the following, we develop an approach to multiple-attribute decision making based on intuitionistic fuzzy Einstein Choquet integral operators. The method involves the following steps:

Step 1. Confirm the fuzzy measure $m\left(c_{j}\right)$ of each attribute $c_{j}(j=1,2, \ldots, n)$. According to Eq. (11), parameter $\rho$ of attributes can be determined, and using Eq. (10), we can obtain all the fuzzy measure $m(A), A \subseteq X$.

Step 2. By Definition $1, r_{i j}$ is reordered such that $r_{i \sigma(1)} \geq r_{i \sigma(2)} \geq \ldots \geq r_{i \sigma(n)}$. Using the IFCA ${ }^{\varepsilon}$ operator:

$$
\begin{aligned}
& r_{i}=\operatorname{IFCA}_{m}^{\varepsilon}\left(r_{i 1}, r_{i 2}, \ldots, r_{i n}\right)= \\
& \left(\frac{\prod_{j=1}^{n}\left(1+\mu_{i \sigma(j)}\right)^{m\left(A_{i \sigma(j)}\right)-m\left(A_{i \sigma(j-1)}\right)}-\prod_{j=1}^{n}\left(1-\mu_{i \sigma(j)}\right)^{m\left(A_{i \sigma(j)}\right)-m\left(A_{i \sigma(j-1)}\right)}}{\prod_{j=1}^{n}\left(1+\mu_{i \sigma(j)}\right)^{m\left(A_{i \sigma(j)}\right)-m\left(A_{i \sigma(j-1)}\right)}+\prod_{j=1}^{n}\left(1-\mu_{i \sigma(j)}\right)^{m\left(A_{i \sigma(j)}\right)-m\left(A_{i \sigma(j-1)}\right)}},\right. \\
& \left.\frac{2 \prod_{j=1}^{n} v_{i \sigma(j)}^{m\left(A_{i \sigma(j)}\right)-m\left(A_{i \sigma(j-1)}\right)}}{\prod_{j=1}^{n}\left(2-v_{i \sigma(j)}\right)^{m\left(A_{i \sigma(j)}\right)-m\left(A_{i \sigma(j-1)}\right)}+\prod_{j=1}^{n} v_{i \sigma(j)}^{m\left(A_{i \sigma(j)}\right)-m\left(A_{i \sigma(j-1)}\right)}}\right),
\end{aligned}
$$


or $\mathrm{IFCG}^{\varepsilon}$ operator:

$$
\begin{aligned}
& r_{i}=\operatorname{IFCG}_{m}^{\varepsilon}\left(r_{i 1}, r_{i 2}, \ldots, r_{i n}\right)= \\
& \left(\frac{2 \prod_{j=1}^{n} \mu_{i \sigma(j)}^{m\left(A_{i \sigma(j)}\right)-m\left(A_{i \sigma(j-1)}\right)}}{\prod_{j=1}^{n}\left(2-\mu_{i \sigma(j)}\right)^{m\left(A_{i \sigma(j)}\right)-m\left(A_{i \sigma(j-1)}\right)}+\prod_{j=1}^{n} \mu_{i \sigma(j)}^{m\left(A_{i \sigma(j)}\right)-m\left(A_{i \sigma(j-1)}\right)}},\right. \\
& \left.\frac{\prod_{j=1}^{n}\left(1+v_{i \sigma(j)}\right)^{m\left(A_{i \sigma(j)}\right)-m\left(A_{i \sigma(j-1)}\right)}-\prod_{j=1}^{n}\left(1-v_{i \sigma(j)}\right)^{m\left(A_{i \sigma(j)}\right)-m\left(A_{i \sigma(j-1)}\right)}}{\prod_{j=1}^{n}\left(1+v_{i \sigma(j)}\right)^{m\left(A_{i \sigma(j)}\right)-m\left(A_{i \sigma(j-1)}\right)}+\prod_{j=1}^{n}\left(1-v_{i \sigma(j)}\right)^{m\left(A_{i \sigma(j)}\right)-m\left(A_{i \sigma(j-1)}\right)}}\right),
\end{aligned}
$$

to get the overall values $r_{i}(i=1,2, \ldots, m)$ of the alternatives $a_{i}(i=1,2, \ldots, m)$, where $(\sigma(1), \sigma(2), \ldots, \sigma(n))$ is a permutation of $(1,2, \ldots, n)$ such that $r_{i \sigma(j-1)} \geq r_{i \sigma(j)}$ for all $j=2,3, \ldots, n$, $A_{i \sigma(j)}=\left\{c_{\sigma(1)}, c_{\sigma(2)}, \ldots, c_{\sigma(j)}\right\}$ and $A_{i \sigma(0)}=\varnothing$.

Step 3. Calculate the scores $S\left(r_{i}\right) \quad(i=1,2, \ldots, m)$ of the collective overall intuitionistic fuzzy preference values $r_{i}(i=1,2, \ldots, m)$ to rank all the alternatives $a_{i} \quad(i=1,2, \ldots, m)$ and then to select the best one(s) (if there is no difference between two scores $S\left(r_{i}\right)$ and $S\left(r_{j}\right)$, then we need to calculate the accuracy degrees $H\left(r_{i}\right)$ and $H\left(r_{j}\right)$ of the collective overall intuitionistic fuzzy preference values $r_{i}$ and $r_{j}$, respectively, and then rank the alternatives $x_{i}$ and $x_{j}$ in accordance with the accuracy degrees $H\left(r_{i}\right)$ and $\left.H\left(r_{j}\right)\right)$.

Step 4. End.

\section{Illustrative example}

Now we consider a decision making problem to support the choice of an alternative to control the degradation of the hydrographic basin of Rio Jaboatão, a river located in the state of the Pernambuco, Brazil. Hydrographic basin committees are the centers of decisions on water resource management in their respective basins (adapted from (Morais, Almeida 2012)). Five criteria (attributes) were considered in order to evaluate the alternatives with regard to the economic, financial, social and environmental aspects, namely (1) $c_{1}$ : investment value, which represents the monetary value of implementing action; (2) $c_{2}$ : maintenance costs, which represents the monetary value of maintaining the action in terms of the annual costs of operation; (3) $c_{3}$ : dependence on third-parties, which evaluates if the efficiency of the action depends on participation of third-parties or needs to consider the involvement of others (society), because it is known that the involvement of third-parties diminishes the effectiveness of actions; (4) $c_{4}$ : industrial impacts, corresponds to the negative impacts that the action will cause on industrial activities from the operational point of view (such as changes to production process); and (5) $c_{5}$ : agricultural impacts, which corresponds to the negative impacts that the action will cause on agricultural activities from the economic (reduction 
of jobs) or legal (fines and fees) points of view. A set of twelve alternatives $a_{i}(i=1,2, \ldots, 12)$ is selected to control environment degradation. Table 1 presents the alternatives and their descriptions. The committees evaluate the twelve alternatives $a_{i}(i=1,2, \ldots, 12)$ in relation to the attributes $c_{j}(j=1,2,3,4,5)$, and construct the decision matrix as listed in Table 2.

Table 1. Alternatives

\begin{tabular}{|c|c|}
\hline Alternative & Description \\
\hline$a_{1}$ & $\begin{array}{l}\text { Secondary sewage treatment in Jaboatão dos Guararapes, which requires that industrial } \\
\text { waste be pre-treated according to the standards laid down. }\end{array}$ \\
\hline$a_{2}$ & $\begin{array}{l}\text { Educational campaigns in the townships within the hydrographic basin (with the exception } \\
\text { of Recife). }\end{array}$ \\
\hline$a_{3}$ & $\begin{array}{l}\text { A campaign with industry to minimize the quantity of water used in production process } \\
\text { by offering monetary incentives for those industries that show positive results. }\end{array}$ \\
\hline$a_{4}$ & $\begin{array}{l}\text { Maintenance of industrial facilities to prevent the water used for refrigeration from being } \\
\text { contaminated by waste matters from industrial processes. }\end{array}$ \\
\hline$a_{5}$ & $\begin{array}{l}\text { To institute policies for controlling the development of new business and/or expansion } \\
\text { of current ones to avoid worsening industrial pollution. }\end{array}$ \\
\hline$a_{6}$ & $\begin{array}{l}\text { Development of a plan of sustainable agriculture specific to the rural producers of Vitória } \\
\text { de Santo Antão which focuses on soil and water conservation for the hydrographic basin } \\
\text { of the Rio Jaboatão. }\end{array}$ \\
\hline$a_{7}$ & Recovery of native vegetation along the banks of the Jaboatão river. \\
\hline$a_{8}$ & $\begin{array}{l}\text { Improving the collection of waste material along the river, such as providing for the } \\
\text { periodic removal of trash. }\end{array}$ \\
\hline$a_{9}$ & Recovery of the natural aquatic ecosystem. \\
\hline$a_{10}$ & $\begin{array}{l}\text { Treatment of the Erosion Points in order to contribute to reducing the silting-up process } \\
\text { in the rivers and in the rainfall drainage network. }\end{array}$ \\
\hline$a_{11}$ & Recuperation of fauna biodiversity. \\
\hline$a_{12}$ & Development of sustainable tourist activities along the Jaboatão river. \\
\hline
\end{tabular}

Table 2. Intuitionistic fuzzy decision matrix $R$

\begin{tabular}{cccccc}
\hline & $c_{1}$ & $c_{2}$ & $c_{3}$ & $c_{4}$ & $c_{5}$ \\
\hline$a_{1}$ & $(0.5,0.4)$ & $(0.5,0.2)$ & $(0.6,0.1)$ & $(0.3,0.2)$ & $(0.7,0.3)$ \\
\hline$a_{2}$ & $(0.5,0.3)$ & $(0.5,0.1)$ & $(0.4,0.3)$ & $(0.4,0.5)$ & $(0.6,0.3)$ \\
\hline$a_{3}$ & $(0.6,0.4)$ & $(0.4,0.3)$ & $(0.5,0.5)$ & $(0.3,0.5)$ & $(0.5,0.2)$ \\
\hline$a_{4}$ & $(0.7,0.2)$ & $(0.7,0.1)$ & $(0.6,0.2)$ & $(0.7,0.3)$ & $(0.8,0.2)$ \\
\hline$a_{5}$ & $(0.8,0.1)$ & $(0.7,0.3)$ & $(0.6,0.1)$ & $(0.7,0.2)$ & $(0.9,0.1)$ \\
\hline$a_{6}$ & $(0.4,0.5)$ & $(0.6,0.1)$ & $(0.6,0.3)$ & $(0.6,0.2)$ & $(0.7,0.3)$ \\
\hline$a_{7}$ & $(0.7,0.3)$ & $(0.6,0.2)$ & $(0.4,0.2)$ & $(0.6,0.3)$ & $(0.5,0.4)$ \\
\hline$a_{8}$ & $(0.4,0.2)$ & $(0.5,0.4)$ & $(0.5,0.1)$ & $(0.5,0.3)$ & $(0.4,0.4)$ \\
\hline$a_{9}$ & $(0.7,0.3)$ & $(0.6,0.3)$ & $(0.8,0.1)$ & $(0.6,0.1)$ & $(0.7,0.2)$ \\
\hline$a_{10}$ & $(0.7,0.3)$ & $(0.7,0.1)$ & $(0.7,0.2)$ & $(0.8,0.1)$ & $(0.8,0.2)$ \\
\hline$a_{11}$ & $(0.6,0.1)$ & $(0.6,0.2)$ & $(0.4,0.6)$ & $(0.5,0.2)$ & $(0.7,0.1)$ \\
\hline$a_{12}$ & $(0.5,0.1)$ & $(0.5,0.2)$ & $(0.7,0.3)$ & $(0.6,0.3)$ & $(0.6,0.4)$ \\
\hline
\end{tabular}


Then, we utilize the proposed procedure to get the most desirable alternative(s).

Step 1. Confirm the importance of each attribute $c_{j}(j=1,2,3,4,5)$, that is, the fuzzy density $m\left(c_{j}\right)$ of each attribute $c_{j}$.

According to the committee opinions, we obtain the fuzzy density of each attribute as:

$m(\varnothing)=0, m\left(\left\{c_{1}\right\}\right)=0.4, m\left(\left\{c_{2}\right\}\right)=0.3, m\left(\left\{c_{3}\right\}\right)=0.2, m\left(\left\{c_{4}\right\}\right)=0.3, m\left(\left\{c_{5}\right\}\right)=0.4$,

By Eq. (11), the $\rho$ of attributes can be determined:

$\rho=-0.75$.

By Eq. (10), we obtain:

$m\left(\left\{c_{1}, c_{2}\right\}\right)=0.61, m\left(\left\{c_{1}, c_{3}\right\}\right)=0.54, m\left(\left\{c_{1}, c_{4}\right\}\right)=0.61, m\left(\left\{c_{1}, c_{5}\right\}\right)=0.68, m\left(\left\{c_{2}, c_{3}\right\}\right)=0.455$, $m\left(\left\{c_{2}, c_{4}\right\}\right)=0.5325, m\left(\left\{c_{2}, c_{5}\right\}\right)=0.61, m\left(\left\{c_{3}, c_{4}\right\}\right)=0.455, m\left(\left\{c_{3}, c_{5}\right\}\right)=0.54, m\left(\left\{c_{4}, c_{5}\right\}\right)=0.61$, $m\left(\left\{c_{1}, c_{2}, c_{3}\right\}\right)=0.7185, m\left(\left\{c_{1}, c_{2}, c_{4}\right\}\right)=0.7728, m\left(\left\{c_{1}, c_{2}, c_{5}\right\}\right)=0.8269, m\left(\left\{c_{1}, c_{3}, c_{4}\right\}\right)=0.7185$, $m\left(\left\{c_{1}, c_{3}, c_{5}\right\}\right)=0.7780, m\left(\left\{c_{1}, c_{4}, c_{5}\right\}\right)=0.8269, m\left(\left\{c_{2}, c_{3}, c_{4}\right\}\right)=0.6527, m\left(\left\{c_{2}, c_{3}, c_{5}\right\}\right)=0.7185$, $m\left(\left\{c_{2}, c_{4}, c_{5}\right\}\right)=0.7728, m\left(\left\{c_{3}, c_{4}, c_{5}\right\}\right)=0.7185, m\left(\left\{c_{1}, c_{2}, c_{3}, c_{4}\right\}\right)=0.8568$,

$m\left(\left\{c_{1}, c_{2}, c_{3}, c_{5}\right\}\right)=0.9029, m\left(\left\{c_{1}, c_{3}, c_{4}, c_{5}\right\}\right)=0.9029, m\left(\left\{c_{1}, c_{2}, c_{4}, c_{5}\right\}\right)=0.9409$,

$m\left(\left\{c_{2}, c_{3}, c_{4}, c_{5}\right\}\right)=0.8568, m\left(\left\{c_{1}, c_{2}, c_{3}, c_{4}, c_{5}\right\}\right)=1$.

Step 2. According to Definition 1, we rearrange the IFV s corresponding to each alternative in descending order. For alternative $a_{1}$, we have:

$$
\begin{aligned}
& r_{1 \sigma(1)}=r_{13}=(0.6,0.1), r_{1 \sigma(2)}=r_{15}=(0.7,0.3), r_{1 \sigma(3)}=r_{12}=(0.5,0.2), r_{1 \sigma(4)}=r_{11}=(0.5,0.4), \\
& r_{1 \sigma(5)}=r_{14}=(0.3,0.2)
\end{aligned}
$$

and

$$
\begin{aligned}
& m\left(\left\{A_{1 \sigma(1)}\right\}\right)=m\left(\left\{c_{3}\right\}\right)=0.2, m\left(\left\{A_{1 \sigma(2)}\right\}\right)=m\left(\left\{c_{3}, c_{5}\right\}\right)=0.54, \\
& m\left(\left\{A_{1 \sigma(3)}\right\}\right)=m\left(\left\{c_{3}, c_{5}, c_{2}\right\}\right)=0.7185, m\left(\left\{A_{1 \sigma(4)}\right\}\right)=m\left(\left\{c_{3}, c_{5}, c_{2}, c_{1}\right\}\right)=0.9029, \\
& m\left(\left\{A_{1 \sigma(5)}\right\}\right)=m\left(\left\{c_{3}, c_{5}, c_{2}, c_{1}, c_{4}\right\}\right)=1 .
\end{aligned}
$$

With the $\mathrm{IFCA}^{\varepsilon}$ operator (i.e. Eq. (50)), we calculate the overall value $r_{1}$ of the alternative $a_{1}$ :

$r_{1}=(0.5803,0.2302)$.

Similarly, we can obtain:

$$
\begin{aligned}
& r_{2}=(0.5169,0.2310), r_{3}=(0.4989,0.3058), r_{4}=(0.7369,0.1820), r_{5}=(0.8213,0.1239), \\
& r_{6}=(0.6098,0.2218), r_{7}=(0.6101,0.2702), r_{8}=(0.4607,0.2383), r_{9}=(0.6991,0.1742), \\
& r_{10}=(0.7651,0.1550), r_{11}=(0.6234,0.1350), r_{12}=(0.5734,0.2)
\end{aligned}
$$


Step 3. Calculate the scores $S\left(r_{i}\right)(i=1,2, \ldots, 12)$ of the collective overall intuitionistic fuzzy preference values $r_{i}(i=1,2, \ldots, 12)$.

$S\left(r_{1}\right)=0.3501, S\left(r_{2}\right)=0.2859, S\left(r_{3}\right)=0.1931, S\left(r_{4}\right)=0.5576, S\left(r_{5}\right)=0.6974, S\left(r_{6}\right)=0.3880$,

$S\left(r_{7}\right)=0.3398, S\left(r_{8}\right)=0.2224, S\left(r_{9}\right)=0.5250, S\left(r_{10}\right)=0.6101, S\left(r_{11}\right)=0.4883, S\left(r_{12}\right)=0.3734$

and thus the ranking of the twelve alternatives $a_{i}(i=1,2, \ldots, 12)$ is:

$$
a_{5} \succ a_{10} \succ a_{4} \succ a_{9} \succ a_{11} \succ a_{6} \succ a_{12} \succ a_{1} \succ a_{7} \succ a_{2} \succ a_{8} \succ a_{3} \text {. }
$$

If we use the IFWG ${ }^{\varepsilon}$ operator (i.e. Eq. (51)) to calculate the overall values corresponding to each alternative, then we obtain:

$$
\begin{aligned}
& r_{1}=(0.5590,0.2536), r_{2}=(0.5106,0.2637), r_{3}=(0.4875,0.3277), r_{4}=(0.7331,0.1910), \\
& r_{5}=(0.7982,0.1349), r_{6}=(0.5973,0.2578), r_{7}=(0.5941,0.2760), r_{8}=(0.4574,0.2652), \\
& r_{9}=(0.6908,0.1916), r_{10}=(0.7604,0.1690), r_{11}=(0.6130,0.1607), r_{12}=(0.5645,0.2258) .
\end{aligned}
$$

And then calculate the scores $S\left(r_{i}\right) \quad(i=1,2, \ldots, 12)$ of the collective overall intuitionistic fuzzy preference values $r_{i}(i=1,2, \ldots, 12)$, we have:

$$
\begin{aligned}
& S\left(r_{1}\right)=0.3053, S\left(r_{2}\right)=0.2469, S\left(r_{3}\right)=0.1598, S\left(r_{4}\right)=0.5420, S\left(r_{5}\right)=0.6633, S\left(r_{6}\right)=0.3395, \\
& S\left(r_{7}\right)=0.3181, S\left(r_{8}\right)=0.1922, S\left(r_{9}\right)=0.4992, S\left(r_{10}\right)=0.5914, S\left(r_{11}\right)=0.4523, S\left(r_{12}\right)=0.3386 .
\end{aligned}
$$

and thus the ranking of the twelve alternatives $a_{i}(i=1,2, \ldots, 12)$ is:

$$
a_{5} \succ a_{10} \succ a_{4} \succ a_{9} \succ a_{11} \succ a_{6} \succ a_{12} \succ a_{7} \succ a_{1} \succ a_{2} \succ a_{8} \succ a_{3} \text {. }
$$

From the above numerical results, we know that the ranking results obtained using the IFCA $^{\varepsilon}$ operator and the IFCG ${ }^{\varepsilon}$ operator are slightly different, but both of the operators produce the same best alternative $a_{5}$.

In the following, the IFCA (i.e. Eq. (12)) operator proposed by Xu (2010) is used to get overall values corresponding to each alternative, we obtain:

$$
\begin{aligned}
& r_{1}=(0.5847,0.2271), r_{2}=(0.5185,0.2267), r_{3}=(0.5015,0.3020), r_{4}=(0.7405,0.1811), \\
& r_{5}=(0.8236,0.1232), r_{6}=(0.6123,0.2173), r_{7}=(0.6133,0.2693), r_{8}=(0.4615,0.2347), \\
& r_{9}=(0.7005,0.1724), r_{10}=(0.7657,0.1538), r_{11}=(0.6254,0.1332), r_{12}=(0.5755,0.1970) .
\end{aligned}
$$

Therefore:

$$
\begin{aligned}
& S\left(r_{1}\right)=0.3576, S\left(r_{2}\right)=0.2917, S\left(r_{3}\right)=0.1995, S\left(r_{4}\right)=0.5595, S\left(r_{5}\right)=0.7004, S\left(r_{6}\right)=0.3949, \\
& S\left(r_{7}\right)=0.3440, S\left(r_{8}\right)=0.2268, S\left(r_{9}\right)=0.5281, S\left(r_{10}\right)=0.6119, S\left(r_{11}\right)=0.4922, S\left(r_{12}\right)=0.3784
\end{aligned}
$$

and thus the ranking of the twelve alternatives $a_{i}(i=1,2, \ldots, 12)$ is:

$$
a_{5} \succ a_{10} \succ a_{4} \succ a_{9} \succ a_{11} \succ a_{6} \succ a_{12} \succ a_{1} \succ a_{7} \succ a_{2} \succ a_{8} \succ a_{3} .
$$


If we use IFCG (i.e. Eq. (13)) operator to get overall values of the alternatives $a_{i}(i=1,2, \ldots, 12)$, we obtain:

$$
\begin{aligned}
& r_{1}=(0.5533,0.2577), r_{2}=(0.5090,0.2694), r_{3}=(0.4846,0.3328), r_{4}=(0.7317,0.1924), \\
& r_{5}=(0.7942,0.1368), r_{6}=(0.5939,0.2649), r_{7}=(0.5897,0.2772), r_{8}=(0.4566,0.2699), \\
& r_{9}=(0.6890,0.1944), r_{10}=(0.7594,0.1712), r_{11}=(0.6102,0.1681), r_{12}=(0.5624,0.2302) .
\end{aligned}
$$

Therefore:

$$
\begin{aligned}
& S\left(r_{1}\right)=0.2956, S\left(r_{2}\right)=0.2396, S\left(r_{3}\right)=0.1518, S\left(r_{4}\right)=0.5394, S\left(r_{5}\right)=0.6574, S\left(r_{6}\right)=0.3290, \\
& S\left(r_{7}\right)=0.3125, S\left(r_{8}\right)=0.1867, S\left(r_{9}\right)=0.4947, S\left(r_{10}\right)=0.5882, S\left(r_{11}\right)=0.4422, S\left(r_{12}\right)=0.3322 .
\end{aligned}
$$

And thus:

$$
a_{5} \succ a_{10} \succ a_{4} \succ a_{9} \succ a_{11} \succ a_{6} \succ a_{12} \succ a_{1} \succ a_{7} \succ a_{2} \succ a_{8} \succ a_{3} .
$$

It is noted that the overall values of the alternatives by the IFCA operator are larger than ones by the IFCA ${ }^{\varepsilon}$ operator, respectively, that is Eq. (35) holds. Similarly, the overall values of the alternatives by the IFCG ${ }^{\varepsilon}$ operator are larger than ones by the IFCG operator, respectively, it also verifies Eq. (49) holds. And the rankings of all the alternatives by IFCA operator and IFCA $^{\varepsilon}$ operator are same, by IFCG operator and IFCG $^{\varepsilon}$ operator are also same. The ranking results obtained using the IFCG operator and $\mathrm{IFCG}^{\varepsilon}$ operator are slightly different. However, the best alternative is same by the four operators. Hence, the alternative $a_{5}$ is most suitable to control the degradation of the hydrographic basin of Rio Jaboatão.

\section{Conclusions}

Being a generalization of fuzzy sets, the IFSs give us an additional possibility to represent imperfect knowledge. This allows us to use more flexible ways to simulate real decision situations. In this paper, we have extended the Einstein operations laws into the intuitionistic fuzzy values, and used the Choquet integral to propose several new aggregation operators of IFSs where interactions phenomena among the decision making criteria are considered. It is shown that the proposed IFCA ${ }^{\varepsilon}$ operator and $\mathrm{IFCG}^{\varepsilon}$ operator generalize several operators, such as IFWA ${ }^{\varepsilon}$ operator, IFA ${ }^{\varepsilon}$ operator, IFOWA ${ }^{\varepsilon}$ operator, IFWOWA ${ }^{\varepsilon}$ operator, $\mathrm{IFWG}^{\varepsilon}$ (Wang, Liu 2011) operator, $\mathrm{IFGA}^{\varepsilon}$ operator, $\mathrm{IFOWG}^{\varepsilon}$ (Wang, Liu 2011) operator, IFWOWG $^{\varepsilon}$ operator. We have also studied some desired properties of the developed operators, such as commutativity, idempotency, boundary, etc. Furthermore, we have studied the relationships between IFCA $^{\varepsilon}$ and IFCA, IFCG $^{\varepsilon}$ and IFCG, respectively. And an approach for multiple-attribute decision making is proposed. Finally, a practical decision making problem involving the water resource management is given to illustrate the multiple attribute decision making process.

In the future, we will investigate the operators to many actual fields, such as architect selection (Keršulienè, Turskis 2011), supply chain planning (Napalkova, Merkuryeva 2012), investment strategy selection (Wu et al. 2012), etc. 


\section{Acknowledgements}

This work was partly supported by the Major Program of the National Social Science Foundation of China (No. 12\&ZD214), the National Natural Science Foundation of China (NSFC) under Grant 71101043, Program for Excellent Talents in Hohai University.

\section{References}

Atanassov, K. 1986. Intuitionistic fuzzy sets, Fuzzy Sets and Systems 20(1): 87-96. http://dx.doi.org/10.1016/S0165-0114(86)80034-3

Atanassov, K. 1999. Intuitionistic fuzzy sets: theory and applications. Heidelberg: Physica-Verlag. 323 p. http://dx.doi.org/10.1007/978-3-7908-1870-3

Beliakov, G.; Bustince, H.; Goswami, D. P.; Mukherjee, U. K.; Pal, N. R. 2011. On averaging operators for Atanassov's intuitionistic fuzzy sets, Information Sciences 181(6): 1116-1124. http://dx.doi.org/10.1016/j.ins.2010.11.024

Bustince, H.; Burillo, P. 1995. Correlation of interval-valued intuitionistic fuzzy sets, Fuzzy Sets and Systems 74(2): 237-244. http://dx.doi.org/10.1016/0165-0114(94)00343-6

Chen, S. M.; Tan, J. M. 1994. Handling multicriteria fuzzy decision-making problems based on vague set theory, Fuzzy Sets and Systems 67(2): 163-172. http://dx.doi.org/10.1016/0165-0114(94)90084-1

Chen, S. M. 1997. Similarity measures between vague sets and between elements, IEEE Transactions on Systems, Man, and Cybernetics 27(1): 153-158. http://dx.doi.org/10.1109/3477.552198

Chen, T. Y. 2007. A note on distances between instuitionistic fuzzy sets and/or interval-valued fuzzy sets based on the Hausdorff metric, Fuzzy Sets and Systems 158(22): 2523-2525. http://dx.doi.org/10.1016/j.fss.2007.04.024

Choquet, G. 1954. Theory of capacities, Annels del Intuitut Fourier 5(1954): 131-295. http://dx.doi.org/10.5802/aif.53

De, S. K.; Biswas, R.; Roy, A. R. 2000. Some operations on intuitionistic fuzzy sets, Fuzzy Sets and Systems 114(3): 477-484. http://dx.doi.org/10.1016/S0165-0114(98)00191-2

Grzegorzewski, P. 2004. Distances between intuitionistic fuzzy sets and/or interval-valued fuzzy sets based on the Hausdorff metric, Fuzzy Sets and Systems 148(2): 319-328. http://dx.doi.org/10.1016/j.fss.2003.08.005

Hong, D. H.; Hwang, S. Y. 1995. Correlation of intuitionistic fuzzy sets in probability spaces, Fuzzy Sets and Systems 75(1): 77-81. http://dx.doi.org/10.1016/0165-0114(94)00330-A

Hong, D. H.; Choi, C. H. 2000. Multicriteria fuzzy decision-making problems based on vague set theory, Fuzzy Sets and Systems 114(1): 103-113. http://dx.doi.org/10.1016/S0165-0114(98)00271-1

Hung, W. L.; Yang, M. S. 2004. Similary measures of intuitionistic fuzzy sets based on Hausdorff distance, Pattern Recognition Letters 25(14): 1603-1611. http://dx.doi.org/10.1016/j.patrec.2004.06.006

Keršulienė, V.; Turskis, Z. 2011. Integrated fuzzy multiple criteria decision making model for architect selection, Technological and Economic Development of Economy 17(4): 645-666.

http://dx.doi.org/10.3846/20294913.2011.635718

Li, D. F.; Cheng, C. T. 2002. New similarity measures of intuitionistic fuzzy sets and application to pattern recognitions, Pattern Recognition Letters 23(1-3): 221-225. http://dx.doi.org/10.1016/S0167-8655(01)00110-6

Li, D. F. 2005. Multiattribute decision making models and methods using intuitionistic fuzzy sets, Journal of Computer and Systems Sciences 70(1): 73-85. http://dx.doi.org/10.1016/j.jcss.2004.06.002 
Liang, Z.; Shi, P. 2003. Similarity measures on intuitionistic fuzzy sets, Pattern Recognition Letters 24(15): 2687-2693. http://dx.doi.org/10.1016/S0167-8655(03)00111-9

Lin, L.; Yuan, X. H.; Xia, Z. Q. 2007. Multicriteria fuzzy decision-making methods based on intuitionistic fuzzy sets, Journal of Computer and Systems Sciences 73(1): 84-88.

http://dx.doi.org/10.1016/j.jcss.2006.03.004

Morais, D. C.; Almeida, A. T. 2012. Group decision making on water resources based on analysis of individual rankings, Omega 40(1): 42-52. http://dx.doi.org/10.1016/j.omega.2011.03.005

Napalkova, L.; Merkuryeva, G. 2012. Multi-objective stochastic simulation-based optimisation applied to supply chain planning, Technological and Economic Development of Economy 18(1): 132-148. http://dx.doi.org/10.3846/20294913.2012.661190

Sugeno, M. 1974. Theory of fuzzy integral and its application: Doctoral Thesis. Tokyo Institute of Technology.

Szmidt, E.; Kacprzyk, J. 2000. Distances between intuitionistic fuzzy sets, Fuzzy Sets and Systems 114(3): 505-518. http://dx.doi.org/10.1016/S0165-0114(98)00244-9

Tan, C. Q.; Chen, X. H. 2010. Intuitionistic fuzzy Choquet operator for multi-criteria decision making, Expert Systems with Applications 37(1): 149-157. http://dx.doi.org/10.1016/j.eswa.2009.05.005

Wang, W. Z.; Liu, X. W. 2011. Intuitionistic fuzzy geometric aggregation operators based on Einstein operations, International Journal of Intelligent Systems 26(11): 1049-1075. http://dx.doi.org/10.1002/int.20498

Wang, W. Z.; Liu, X. W. 2012. Intuitionistic fuzzy information aggregation using Einstein operations, IEEE Transactions on Fuzzy Systems 20(5): 923-938. http://dx.doi.org/10.1109/TFUZZ.2012.2189405

Wang, Z.; Klir, G. 1992. Fuzzy measure theory. New York: Plenum Press. 354 p. http://dx.doi.org/10.1007/978-1-4757-5303-5

Wei, G. W. 2009. Some geometric aggregation functions and their application to dynamic multiple attribute decision making in the intuitionistic fuzzy setting, International Journal of Uncertainty, Fuzziness and Knowledge-Based Systems 17(2): 179-196.

http://dx.doi.org/10.1142/S0218488509005802

Wei, G. W. 2010. Some induced geometric aggregation operators with intuitionistic fuzzy information and their application to group decision making, Applied Soft Computing 10(2): 423-431.

http://dx.doi.org/10.1016/j.asoc.2009.08.009

Wu, W. S.; Kou, G.; Peng, Y.; Ergu, D. 2012. Improved AHP-group decision making for investment strategy selection, Technological and Economic Development of Economy 18(2): 299-316. http://dx.doi.org/10.3846/20294913.2012.680520

Xu, Y. J.; Huang, C.; Da, Q. L.; Liu, X. W. 2010. Linear goal programming approach to obtaining the weights of intuitionistic fuzzy ordered weighted averaging operator, Journal of Systems Engineering and Electronics 21(6): 990-994.

Xu, Y. J.; Wang, H. M. 2012. The induced generalized aggregation operators for intuitionistic fuzzy sets and their application in group decision making, Applied Soft Computing 12(3): 1168-1179. http://dx.doi.org/10.1016/j.asoc.2011.11.003

Xu, Z. S.; Da, Q. L. 2002. The ordered weighted geometric averaging operators, International Journal of Intelligent Systems 17(7): 709-716. http://dx.doi.org/10.1002/int.10045

Xu, Z. S.; Yager, R. R. 2006. Some geometric aggregation operators based on intuitionistic fuzzy sets, International Journal of General Systems 35(4): 417-433. http://dx.doi.org/10.1080/03081070600574353

Xu, Z. S. 2007. Intuitionistic fuzzy aggregation operators, IEEE Transations on Fuzzy Systems 15(6): 1179-1187. http://dx.doi.org/10.1109/TFUZZ.2006.890678

$\mathrm{Xu}, \mathrm{Z}$. S. 2010. Choquet integrals of weighted intuitionistic fuzzy information, Information Sciences 180(5): 726-736. http://dx.doi.org/10.1016/j.ins.2009.11.011 
$\mathrm{Xu}, \mathrm{Z}$. S. 2011. Approaches to multiple attribute group decision making based on intuitionistic fuzzy power aggregation operators, Knowledge-Based Systems 24(6): 749-760. http://dx.doi.org/10.1016/j.knosys.2011.01.011

Yager, R. R. 1988. On ordered weighted averaging aggregation operators in multicirteria decision making, IEEE Transactions on Systems, Man, and Cybernetics 18(1): 183-190. http://dx.doi.org/10.1109/21.87068

Zadeh, L. A. 1965. Fuzzy sets, Information and Control 8(3): 338-353. http://dx.doi.org/10.1016/S0019-9958(65)90241-X

Zhao, H.; Xu, Z. S.; Ni, M. F.; Liu, S. S. 2010. Generalized aggregation operators for intuitionistic fuzzy sets, International Journal of Intelligent Systems 25(1): 1-30. http://dx.doi.org/10.1002/int.20386

Yejun XU was born in 1979. He received the MS degree in 2005 and the PhD degree in 2009 both in Management Science and Engineering, both from Southeast University, China. Currently he is an Associate Professor with Business School, Hohai University. He has contributed more than 40 articles to professional journals, such as Information Sciences, International Journal of Approximate Reasoning, Knowledge-Based Systems, etc. His current research interests include information fusion, group decision making under uncertainty.

Huimin WANG was born in 1963. She is now a Professor with State Key Laboratory of Hydrology-Water Resources and Hydraulic Engineering, Hohai University, and also a Professor with Business School, Hohai University. She has contributed over 150 articles to professional journals. Her research interests include water resource management, management science and system engineering.

José M. MERIGÓ is a Senior Research Fellow at the University of Manchester, UK. He has a MSc and a PhD degree in Business Administration from University of Barcelona, Spain. He also holds a Bachelor's Degrees in Economics and a Master's Degree in European Business Administration and Business Law from Lund University, Sweden. He has published more than 200 papers in journals, books and conference proceedings. He has published 9 books including two edited with World Scientific and three with Springer. $\mathrm{He}$ is on the editorial board of several journals. He is one of the main editors of the International Journal of Management Science and Information Technology. He has participated in several scientific committees and serves as a reviewer in a wide range of journals. He is currently interested in aggregation operators, decision making, bibliometrics and uncertainty. 